MATHEMATICS OF COMPUTATION

Volume 74, Number 252, Pages 1599-1619

S 0025-5718(05)01743-6

Article electronically published on January 3, 2005

\title{
RELIABLE A POSTERIORI ERROR CONTROL FOR NONCONFORMING FINITE ELEMENT APPROXIMATION OF STOKES FLOW
}

\author{
W. DÖRFLER AND M. AINSWORTH
}

\begin{abstract}
We derive computable a posteriori error estimates for the lowest order nonconforming Crouzeix-Raviart element applied to the approximation of incompressible Stokes flow. The estimator provides an explicit upper bound that is free of any unknown constants, provided that a reasonable lower bound for the inf-sup constant of the underlying problem is available. In addition, it is shown that the estimator provides an equivalent lower bound on the error up to a generic constant.
\end{abstract}

\section{INTRODUCTION}

1.1. Setting. The use of solenoidal (i.e., divergence-free) finite elements is attractive since it allows the decoupling of the velocity from the pressure in incompressible flow calculations. It is well known that the only conforming solenoidal finite elements are based on high degree polynomials, and therefore, if one is to avoid high degree finite elements, then one is obliged to use nonconforming elements. The lowest order such element is the $\mathbb{P}^{1}-\mathbb{P}^{0}$ element (for the velocity and pressure components, respectively), developed by Crouzeix and Raviart 9. For an overview of this and related methods, see [5, Ch. IV.3]. We point out that the analysis of this element is confined to problems with pure Dirichlet boundary conditions due to its failure to satisfy a discrete Korn's inequality, although there is evidence that the method is of practical use in spite of this apparent failing [23. Furthermore, the element is widely used in the numerical simulation of Stokes, Navier-Stokes and non-Newtonian flow [20, 3, 13, 22, 24, 4].

Adaptive methods for nonconforming elements have been studied for some time now, and a posteriori error estimates have been obtained that bound the true error above and below. Early papers treated the case of the linear (and nonlinear) Poisson problem 1, 14, 12, 19], and estimators for the Stokes problem have been obtained, for example, in [26, 10, 7]. Maximum norm error estimators were derived in [11. However, such estimates almost always contain generic (unknown) constants and as such do not provide actual computable error bounds. Of course, it is possible to

Received by the editor November 17, 2003 and, in revised form, August 7, 2004.

2000 Mathematics Subject Classification. Primary 65N12, 65N15, 65N30.

Key words and phrases. Computable error bounds, a posteriori error estimates, nonconforming finite elements, Stokes flow.

This work was initiated during the authors' visit to the Newton Institute for Mathematical Sciences in Cambridge. The support of the second author by the Leverhulme Trust under a Leverhulme Trust Fellowship is gratefully acknowledged. 
attempt to evaluate the constants appearing in the error bounds [6] but this kind of approach generally leads to overly pessimistic estimates. An alternative approach is to attempt to estimate the error in functional outputs, as in [20].

It was observed in [2] that computable upper bounds can be derived for nonconforming elements where such unknown constants are absent. Moreover, the bounds are found to be not only accurate, but also easy and cheap to compute. We adopt a similar approach here to derive constant-free (for the upper bound) a posteriori error estimates for the Crouzeix-Raviart finite element approximation of Stokes flow provided that a lower bound for the inf-sup constant for the continuous problem is available.

The argument is presented in full detail for the case of homogeneous Dirichlet boundary conditions in two space dimensions. We also outline the modifications needed to generalize to nonhomogeneous data in planar domains and to the extensions to the three dimensional setting.

Notation. Throughout, we use standard notation for the Lebesgue $\left(L^{p}(G)^{k}\right)$ and Sobolev $\left(H^{m}(G)^{k}\right)$ spaces on some open domain $G \subset \mathbb{R}^{d}$ into $\mathbb{R}^{k}$ for $d, k \in \mathbb{N}$ and $p \in[1, \infty]$. The norm on $L^{2}(G)^{k}$ is denoted by $\|\cdot\|_{G}$. For vector valued functions $\boldsymbol{v}=\left[v_{i}\right]_{i=1, d}: G \rightarrow \mathbb{R}^{d}$ we let $\boldsymbol{\nabla} \boldsymbol{v} \equiv \operatorname{grad}(\boldsymbol{v}):=\left[\partial_{j} v_{i}\right]_{i, j=1, d}, \boldsymbol{\nabla} \cdot \boldsymbol{v} \equiv$ $\operatorname{div}(\boldsymbol{v}):=\sum_{i=1}^{d} \partial_{i} v_{i}$. In particular, for the two dimensional case, $d=2$, we define $\nabla^{\perp}:=\left[\partial_{2},-\partial_{1}\right]$ so that $\nabla^{\perp} s=\left[\partial_{2} s,-\partial_{1} s\right]$ for scalar functions $s: G \rightarrow \mathbb{R}$ and $\boldsymbol{\nabla}^{\perp} \cdot \boldsymbol{v} \equiv \operatorname{curl}_{2}(\boldsymbol{v})=\partial_{2} v_{1}-\partial_{1} v_{2}$. Likewise, for matrix valued functions $\boldsymbol{A}: G \rightarrow \mathbb{R}^{d, d}$ we let $\boldsymbol{\nabla} \cdot \boldsymbol{A}$ be the vector field $\left[\sum_{j=1}^{d} \partial_{j} A_{i j}\right]_{i=1, d}$.

1.2. The Stokes problem. Let $\Omega \subset \mathbb{R}^{d}, d \in\{2,3\}$, be an open polygonal or polyhedral domain. For a given source $\boldsymbol{f} \in L^{2}(\Omega)^{d}$, we seek a velocity field $\boldsymbol{u}: \Omega \rightarrow$ $\mathbb{R}^{d}$ and a pressure $p: \Omega \rightarrow \mathbb{R}$ satisfying the Stokes equations,

$$
\begin{aligned}
-\Delta \boldsymbol{u}+\boldsymbol{\nabla} p=\boldsymbol{f} & \text { in } \Omega, \\
\boldsymbol{\nabla} \cdot \boldsymbol{u}=0 & \text { in } \Omega, \\
\boldsymbol{u}=\mathbf{0} & \text { on } \partial \Omega,
\end{aligned}
$$

subject to the side constraint $\int_{\Omega} p=0$. Initially, we confine our attention to homogeneous boundary conditions but this assumption is relaxed in Section 4 .

The weak form of the Stokes problem consists of seeking $\boldsymbol{u} \in \boldsymbol{V}$ and $p \in M$ satisfying

$$
\int_{\Omega}\{\boldsymbol{\nabla} \boldsymbol{u}: \boldsymbol{\nabla} \boldsymbol{v}-p \boldsymbol{\nabla} \cdot \boldsymbol{v}+\boldsymbol{\nabla} \cdot \boldsymbol{u} q\}=\int_{\Omega} \boldsymbol{f} \cdot \boldsymbol{v} \quad \forall \boldsymbol{v} \in \boldsymbol{V}, \forall q \in M,
$$

where

$$
\boldsymbol{V}:=H_{0}^{1}(\Omega)^{d}, \quad M:=L_{0}^{2}(\Omega):=\left\{q \in L^{2}(\Omega): \int_{\Omega} q=0\right\} .
$$

If the inf-sup condition

$$
\inf _{q \in M} \sup _{\boldsymbol{v} \in \boldsymbol{V}} \frac{\int_{\Omega} \boldsymbol{\nabla} \cdot \boldsymbol{v} q}{\|\boldsymbol{\nabla} \boldsymbol{v}\|\|q\|} \geq c_{0}>0
$$

holds for some positive constant $c_{0}$ depending on the domain $\Omega$ and the norm $\|\cdot\| \equiv\|\cdot\|_{\Omega}$, then it may be shown (see [5, Ch. II.1] or [16, Ch. IV]) that there 
exists a unique solution of problem (1.2) which depends continuously on the data

$$
\|\boldsymbol{\nabla} \boldsymbol{u}\|+c_{0}\|p\| \leq C(\Omega, d)\|\boldsymbol{f}\| .
$$

The mapping

$$
[\boldsymbol{u}, \boldsymbol{v}] \mapsto \int_{\Omega} \boldsymbol{\nabla} \boldsymbol{u}: \nabla \boldsymbol{v}
$$

defines a positive definite bilinear form on the closed subspace $\boldsymbol{X} \subset \boldsymbol{V}$ defined by

$$
\boldsymbol{X}:=\left\{\boldsymbol{v} \in H_{0}^{1}(\Omega)^{d}: \nabla \cdot \boldsymbol{v}=0\right\}
$$

As a matter of fact, this property may be exploited to reformulate the problem whereby we seek $\boldsymbol{u} \in \boldsymbol{X}$ such that

$$
\int_{\Omega} \boldsymbol{\nabla} \boldsymbol{u}: \nabla \boldsymbol{v}=\int_{\Omega} \boldsymbol{f} \cdot \boldsymbol{v} \quad \forall \boldsymbol{v} \in X .
$$

One advantage of reformulating the problem over the space $\boldsymbol{X}$ is that the pressure $p$ does not appear explicitly and the problem for the velocity field is positive definite; although on the other hand, it is more difficult to discretise the subspace $\boldsymbol{X}$.

\section{The nonconforming Crouzeix-Raviart Element}

2.1. Triangulation. Let $\mathcal{K}$ be a triangulation of the domain $\Omega \subset \mathbb{R}^{2}$. We assume that $\bar{\Omega}=\bigcup_{K \in \mathcal{K}} K$ and that the nonempty intersection of distinct elements is either a single common edge or vertex of both elements. In addition, the triangles are supposed to be shape regular in the sense that the usual minimal angle condition holds uniformly over all sequences of triangulations. Observe that the triangulation need not necessarily be quasi-uniform, although the assumptions do mean that the triangulation will be locally quasi-uniform.

Let $\overline{\mathcal{N}}$ be a set indexing the element vertices, the subset $\mathcal{N}$ indexes vertices on the interior of the domain, while $\mathcal{N}^{\partial}$ indexes vertices on the domain boundary. The centroid and area of an element $K \in \mathcal{K}$ are denoted by $\boldsymbol{x}_{K}$ and $|K|$, respectively. The piecewise constant function defined by $h(\boldsymbol{x}):=h_{K}$ for $\boldsymbol{x} \in K$, where $h_{K}:=$ $\operatorname{diam}(K)$, is used to define the mesh-size $h$ of $\mathcal{K}$. The vector $\boldsymbol{n}_{K}$ denotes the unit exterior normal on the boundary $\partial K$ and $\boldsymbol{t}_{K}:=\boldsymbol{n}_{K}^{\perp}$ denotes the unit tangent vector, obtained by a $90^{\circ}$ rotation of $\boldsymbol{n}_{K}$ in an anti-clockwise sense. We denote the set of all element edges by $\overline{\mathcal{E}}$, the set of interior edges by $\mathcal{E}$, and the set of boundary edges by $\mathcal{E}^{\partial}$. The midpoint and diameter of an edge $\gamma$ are denoted by $\boldsymbol{x}_{\gamma}$ and $h_{\gamma}$, respectively. Each edge $\gamma \in \overline{\mathcal{E}}$ is oriented by assigning a unit normal vector $\boldsymbol{n}_{\gamma}$ along with a corresponding unit tangent vector $\boldsymbol{t}_{\gamma}:=\boldsymbol{n}_{\gamma}^{\perp}$. In the case of an exterior edge $\gamma \in \mathcal{E}^{\partial}$ the vector $\boldsymbol{n}_{\gamma}$ is always taken to be the exterior normal on $\partial \Omega$. Observe that these definitions mean that $\boldsymbol{n}_{K ; \gamma}= \pm \boldsymbol{n}_{\gamma}$ and $\boldsymbol{t}_{K ; \gamma}= \pm \boldsymbol{t}_{\gamma}$, and that the positive sign is always taken in the case of a boundary edge $\gamma \subset \partial \Omega$.

It is useful to introduce broken spaces relative to a triangulation $\mathcal{K}$. For example, the space $H^{1}(\mathcal{K}):=\bigotimes_{K \in \mathcal{K}} H^{1}(K)$ consists of functions whose restriction to individual elements are locally $H^{1}$-smooth, but which are in general discontinuous across element interfaces. Likewise, $\mathbb{P}^{l}(\mathcal{K})$ denotes the space of piecewise polynomials of degree at most $l \in \mathbb{N}$ with respect to the triangulation $\mathcal{K}$. The subspace $\mathbb{P}^{l}(\mathcal{K}) \cap H^{1}(\Omega)$ consisting of continuous piecewise polynomials is denoted by $\mathbb{P}_{\mathrm{C}}^{l}(\mathcal{K})$. 
Although functions in broken spaces are not differentiable at element boundaries, it is possible to define an elementwise gradient $\nabla_{h}$ as follows:

$$
\nabla_{h} w(\boldsymbol{x}):=\boldsymbol{\nabla} w(\boldsymbol{x}), \quad \boldsymbol{x} \in \operatorname{int}(K), \quad \forall K \in \mathcal{K} .
$$

On an interior edge $\gamma=\partial K \cap \partial K^{\prime}$, the jump in the trace of $w \in H^{1}(\mathcal{K})$ across the edge is denoted by $[w]_{\gamma}:=w_{K ; \gamma}-w_{K^{\prime} ; \gamma}$, where the elements are ordered so that the edge normal $\boldsymbol{n}_{\gamma}$ points from $K^{\prime}$ into $K$. Equally well, for a sufficiently smooth function $w,\left[\partial_{\boldsymbol{t}} w\right]_{\gamma}$ and $\left[\partial_{\boldsymbol{n}} w\right]_{\gamma}$ denote jumps in traces of derivatives of $w$ across an interior edge, where $\partial_{\boldsymbol{t}}:=\boldsymbol{t}_{\gamma} \cdot \boldsymbol{\nabla}$ and $\partial_{\boldsymbol{n}}:=\boldsymbol{n}_{\gamma} \cdot \boldsymbol{\nabla}$ denote the normal and tangential derivatives.

2.2. The finite element space. The finite element discretisation of problem (1.2) is defined in terms of a pair of subspaces $\boldsymbol{V}_{h} \subset \boldsymbol{V}$ and $M_{h} \subset M$, consisting of piecewise polynomials relative to the triangulation $\mathcal{K}$ of the domain $\Omega$. In order for the discrete problem

$$
\begin{aligned}
& \int_{\Omega}\left\{\nabla_{h} \boldsymbol{u}_{h}: \nabla_{h} \boldsymbol{v}_{h}-p_{h} \boldsymbol{\nabla}_{h} \cdot \boldsymbol{v}_{h}+\nabla_{h} \cdot \boldsymbol{u}_{h} q_{h}\right\}=\int_{\Omega} \boldsymbol{f} \cdot \boldsymbol{v}_{h} \\
& \forall \boldsymbol{v}_{h} \in \boldsymbol{V}_{h} \forall q_{h} \in M_{h}
\end{aligned}
$$

to be uniquely solvable, it is necessary for the pair $\boldsymbol{V}_{h}, M_{h}$ to be properly balanced so that a discrete version of the inf-sup condition (1.3) is satisfied [5, p. 78]. Not all combinations give rise to a stable approximation. For instance, the lowest order combination $\boldsymbol{V}_{h} \subset \mathbb{P}_{\mathrm{C}}^{1}(\mathcal{K})^{2}$ and $M_{h} \subset \mathbb{P}^{0}(\mathcal{K})$ does not satisfy an inf-sup condition [5. p. 208].

Given a stable combination, the direct approach leads to a saddle point problem coupling the unknowns in the discrete velocity and pressure. Alternatively, by analogy with the approach involving the divergence-free subspace $\boldsymbol{X}$ described in Section 11 one can obtain a symmetric positive definite system involving only the discrete velocity, through the introduction of the subspace $\boldsymbol{X}_{h} \subset \boldsymbol{V}_{h}$ consisting of discretely divergence-free functions. At first sight, such an approach has much to commend it. Unfortunately, it has been found [15] that the only nontrivial divergence-free conforming polynomials are of high order. Nevertheless, if one is prepared to relax the requirement for a conforming approximation of the velocity field and instead use a nonconforming scheme (details later), then it is even possible to use the lowest order approximation whereby $\boldsymbol{V}_{h} \subset \mathbb{P}^{1}(\mathcal{K})^{2}$ and $M_{h} \subset \mathbb{P}^{0}(\mathcal{K})$. The extra freedom in the velocity means that this combination is stable in the sense that a discrete inf-sup condition is now satisfied, despite the fact that this fails to hold for the corresponding conforming combination. More importantly, it is possible to construct the subspace $\boldsymbol{X}_{h} \subset \boldsymbol{V}_{h}$ consisting of piecewise divergence-free functions explicitly.

Let $\theta_{\gamma} \in \mathbb{P}^{1}(\mathcal{K})$ be the first order scalar, nonconforming function uniquely defined by the conditions $\theta_{\gamma}\left(\boldsymbol{x}_{\gamma}\right)=\delta_{\gamma \gamma^{\prime}}$ for $\gamma, \gamma^{\prime} \in \overline{\mathcal{E}}$. These functions are nonconforming in the sense that they fail to belong to the space $H^{1}(\Omega)$ since their traces are discontinuous across element interfaces. Nevertheless, such functions are continuous at edge midpoints and therefore satisfy a weakened conformity condition whereby the average values of the traces are continuous at element interfaces: $\int_{\gamma} v_{h ; K}=$ $\int_{\gamma} v_{h ; K^{\prime}}$ on $\gamma=K \cap K^{\prime}$. The finite element spaces for the approximation of the 
Stokes problem are then defined by

$$
\boldsymbol{V}_{h}:=\operatorname{span}\left\{\boldsymbol{\tau}_{\gamma}, \boldsymbol{\nu}_{\gamma}: \gamma \in \mathcal{E}\right\}, \quad M_{h}:=\left\{q \in \mathbb{P}^{0}(\mathcal{K}): \int_{\Omega} q=0\right\},
$$

where $\boldsymbol{\tau}_{\gamma}:=\theta_{\gamma} \boldsymbol{t}_{\gamma}$ and $\boldsymbol{\nu}_{\gamma}:=\theta_{\gamma} \boldsymbol{n}_{\gamma}$. Evidently, the space $\boldsymbol{V}_{h}$ is nonconforming in the sense described above. The stability and convergence of this method are studied in the original paper of Crouzeix and Raviart 9 .

Let $\boldsymbol{X}_{h}$ denote the subspace consisting of piecewise divergence-free functions,

$$
\boldsymbol{X}_{h}:=\operatorname{span}\left\{\boldsymbol{v}_{h} \in \boldsymbol{V}_{h}: \nabla_{h} \cdot \boldsymbol{v}_{h}=0\right\} .
$$

Owing to the lack of conformity, $\boldsymbol{X}_{h}$ is not a subspace of $\boldsymbol{X}$ given by (1.5). A basis for $\boldsymbol{X}_{h}$ can be defined explicitly as follows. For $n \in \mathcal{N}$, let

$$
\boldsymbol{\rho}_{n}:=\sum_{\gamma \in \mathcal{E}: \boldsymbol{x}_{n} \in \gamma} \frac{\sigma_{n ; \gamma}}{h_{\gamma}} \boldsymbol{\nu}_{\gamma}
$$

where $\sigma_{n ; \gamma} \in\{ \pm 1\}$ is chosen so that the vectors $\sigma_{n ; \gamma} \boldsymbol{n}_{\gamma}$ defined on element edges trace a path around the node $\boldsymbol{x}_{n}$ in an anti-clockwise sense. The subspace $\boldsymbol{X}_{h} \subset \boldsymbol{V}_{h}$ is then given by

$$
\boldsymbol{X}_{h}=\operatorname{span}\left\{\boldsymbol{\tau}_{\gamma}, \boldsymbol{\rho}_{n}: \gamma \in \mathcal{E}, n \in \mathcal{N}\right\} .
$$

In order to see this, first note that every $\boldsymbol{v}_{h} \in \boldsymbol{X}_{h}$ satisfies $0=\oint_{\partial K} \boldsymbol{v}_{h} \cdot \boldsymbol{n}_{K}=$ $\int_{K} \boldsymbol{\nabla} \cdot \boldsymbol{v}_{h}$, by (2.3), and hence, since $\boldsymbol{v}_{h}$ is linear, it follows that $\boldsymbol{\nabla} \cdot \boldsymbol{v}_{h}=0$ on $K$. As intimated earlier, the availability of an explicit representation for the subspace $\boldsymbol{X}_{h}$ means that it is possible to decouple the computation of the discrete velocity from the pressure field. The discrete velocity field $\boldsymbol{u}_{h} \in \boldsymbol{X}_{h}$ is uniquely determined by the condition

$$
\int_{\Omega} \nabla_{h} \boldsymbol{u}_{h}: \nabla_{h} \boldsymbol{v}_{h}=\int_{\Omega} \boldsymbol{f} \cdot \boldsymbol{v}_{h} \quad \forall \boldsymbol{v}_{h} \in \boldsymbol{X}_{h}
$$

Once the discrete velocity is in hand, the corresponding pressure $p_{h}$ can be computed, without having to solve a linear system, using a marching procedure over the triangulation as described in [17.

2.3. A projection operator. Let $\boldsymbol{v} \in \boldsymbol{V}$ be given. Then the conditions

$$
\boldsymbol{\Pi}_{h} \boldsymbol{v}\left(\boldsymbol{x}_{\gamma}\right):=f_{\gamma} \boldsymbol{v} \quad \forall \gamma \in \overline{\mathcal{E}}
$$

define a unique function $\boldsymbol{\Pi}_{h} \boldsymbol{v} \in \boldsymbol{V}_{h}$. This rule defines a nonconforming projection operator $\boldsymbol{\Pi}_{h}: \boldsymbol{V} \rightarrow \boldsymbol{V}_{h}$. Moreover, the operator has the property $\boldsymbol{\Pi}_{h}: \boldsymbol{X} \rightarrow \boldsymbol{X}_{h}$. To see this, first observe that the above conditions imply that $\int_{\gamma} \boldsymbol{\Pi}_{h} \boldsymbol{v} \cdot \boldsymbol{n}_{K}=$ $\int_{\gamma} \boldsymbol{v} \cdot \boldsymbol{n}_{K}$ for all $\gamma \in \overline{\mathcal{E}}$. Therefore, if $\boldsymbol{v} \in \boldsymbol{X}$, then

$$
\oint_{\partial K} \boldsymbol{\Pi}_{h} \boldsymbol{v} \cdot \boldsymbol{n}_{K}=\oint_{\partial K} \boldsymbol{v} \cdot \boldsymbol{n}_{K}=\int_{K} \boldsymbol{\nabla} \cdot \boldsymbol{v}=0,
$$

and hence $\boldsymbol{\Pi}_{h} \boldsymbol{v} \in \boldsymbol{X}_{h}$. Finally, the projection $\boldsymbol{\Pi}_{h}$ satisfies the following local interpolation estimate (see, e.g., [2]):

$$
\left\|\boldsymbol{v}-\boldsymbol{\Pi}_{h} \boldsymbol{v}\right\|_{K}+h_{K}\left\|\boldsymbol{\nabla}\left(\boldsymbol{v}-\boldsymbol{\Pi}_{h} \boldsymbol{v}\right)\right\|_{K} \leq C h_{K}\|\boldsymbol{\nabla} \boldsymbol{v}\|_{K} \quad \forall K \in \mathcal{K} \quad \forall \boldsymbol{v} \in \boldsymbol{V} .
$$


2.4. Enhanced approximation space. For later purposes we will embed $\boldsymbol{V}_{h} \times$ $M_{h}$ into a pair of enhanced (higher order) conforming spaces $\boldsymbol{V}_{h}^{*} \subset \boldsymbol{V}$ and $M_{h}^{*} \subset M$. Roughly speaking, the enhanced velocity space $\boldsymbol{V}_{h}^{*}$ consists of continuous piecewise quadratic functions augmented by so-called cubic "bubble" functions associated with the element interiors, while the enhanced pressure space $M_{h}^{*}$ consists of continuous piecewise linear functions. The precise details are as follows.

Given an edge $\gamma \in \overline{\mathcal{E}}$, let $\theta_{\gamma}^{*}$ denote the conforming piecewise quadratic function defined uniquely by the conditions $f_{\gamma^{\prime}} \theta_{\gamma}^{*}=\delta_{\gamma \gamma^{\prime}}$ and $\theta_{\gamma}^{*}\left(\boldsymbol{x}_{n}\right)=0$ for all $n \in \mathcal{N}$. Analogously to Section 2.2 we define

$$
\boldsymbol{\tau}_{\gamma}^{*}:=\theta_{\gamma}^{*} \boldsymbol{t}_{\gamma}, \quad \boldsymbol{\nu}_{\gamma}^{*}:=\theta_{\gamma}^{*} \boldsymbol{n}_{\gamma}, \quad \boldsymbol{\rho}_{n}^{*}:=\sum_{\gamma \in \mathcal{E}: \boldsymbol{x}_{n} \in \gamma} \frac{\sigma_{n ; \gamma}}{h_{\gamma}} \boldsymbol{\nu}_{\gamma}^{*} .
$$

For each node $n \in \mathcal{N}$, let $\phi_{n}^{*}$ be the conforming piecewise quadratic function uniquely defined by the conditions $\phi_{n}^{*}\left(\boldsymbol{x}_{m}\right):=\delta_{n m}$ and $\int_{\gamma} \phi_{n}^{*}=0$ for all $\gamma \in \overline{\mathcal{E}}$. Finally, let $\beta_{K}^{*}$ be the piecewise cubic function supported on element $K$, whose value at the centroid $\boldsymbol{x}_{K}$ is unity.

The enhanced spaces are defined by

$$
\begin{aligned}
& \boldsymbol{V}_{h}^{*}:=\operatorname{span}\left\{\boldsymbol{\tau}_{\gamma}^{*}, \boldsymbol{\nu}_{\gamma}^{*}, \phi_{n}^{*} \hat{\boldsymbol{e}}_{m}, \beta_{K}^{*} \hat{\boldsymbol{e}}_{m}: \gamma \in \mathcal{E}, n \in \mathcal{N}, m \in\{1,2\}, K \in \mathcal{K}\right\}, \\
& M_{h}^{*}:=\left\{q_{h} \in \mathbb{P}_{\mathrm{C}}^{1}(\mathcal{K}): \int_{\Omega} q_{h}=0\right\},
\end{aligned}
$$

where $\hat{\boldsymbol{e}}_{1}$ and $\hat{\boldsymbol{e}}_{2}$ denote the unit basis vectors for the Euclidean space $\mathbb{R}^{2}$. As before, the associated subspace $\boldsymbol{X}_{h}^{*} \subset \boldsymbol{V}_{h}^{*}$, defined by

$$
\boldsymbol{X}_{h}^{*}:=\left\{\boldsymbol{v}_{h} \in \boldsymbol{V}_{h}^{*}: \int_{\Omega} \boldsymbol{\nabla} \cdot \boldsymbol{v}_{h} q_{h}=0 \quad \forall q_{h} \in M_{h}^{*}\right\},
$$

has an important role to play. The resulting finite element space $\boldsymbol{X}_{h}^{*}$ may be regarded as an enriched Taylor-Hood space [5, Ch. 6.3].

\section{A posteriori ERROR ESTIMATES FOR HOMOGENEOUS BOUNDARY DATA}

For simplicity, we begin by considering the case of homogeneous boundary conditions and defer the treatment of the general case until later.

3.1. Error representation. Let $\boldsymbol{e}:=\boldsymbol{u}-\boldsymbol{u}_{h} \in \boldsymbol{X}+\boldsymbol{X}_{h}$ and $e_{p}:=p-p_{h} \in L_{0}^{2}(\Omega)$ denote the error in the velocity and the pressure, respectively. Thanks to (1.2) and (2.1), these quantities satisfy

$$
\int_{\Omega}\left\{\boldsymbol{\nabla}_{h} \boldsymbol{e}: \boldsymbol{\nabla} \boldsymbol{v}-e_{p} \boldsymbol{\nabla} \cdot \boldsymbol{v}\right\}=\int_{\Omega}\left\{\boldsymbol{f} \cdot \boldsymbol{v}-\boldsymbol{\nabla}_{h} \boldsymbol{u}_{h}: \boldsymbol{\nabla} \boldsymbol{v}+p_{h} \boldsymbol{\nabla} \cdot \boldsymbol{v}\right\} \quad \forall \boldsymbol{v} \in \boldsymbol{V} .
$$

Lemma 1. Let $\boldsymbol{\Pi}_{h}: \boldsymbol{V} \rightarrow \boldsymbol{V}_{h}$ be the nonconforming projection operator defined in Section 2.3. Then,

$$
\int_{\Omega}\left\{\nabla_{h} e: \nabla \boldsymbol{v}-e_{p} \boldsymbol{\nabla} \cdot \boldsymbol{v}\right\}=\int_{\Omega} \boldsymbol{f} \cdot\left(\boldsymbol{v}-\boldsymbol{\Pi}_{h} \boldsymbol{v}\right) \quad \forall \boldsymbol{v} \in \boldsymbol{V} .
$$


Proof. With the aid of (3.1) and (2.1),

$$
\begin{aligned}
\int_{\Omega}\left\{\nabla_{h} \boldsymbol{e}\right. & \left.: \boldsymbol{\nabla} \boldsymbol{v}-e_{p} \boldsymbol{\nabla} \cdot \boldsymbol{v}\right\}=\int_{\Omega}\left\{\boldsymbol{f} \cdot \boldsymbol{v}-\boldsymbol{\nabla} \boldsymbol{u}_{h}: \boldsymbol{\nabla} \boldsymbol{v}+p_{h} \boldsymbol{\nabla} \cdot \boldsymbol{v}\right\} \\
& =\int_{\Omega}\left\{\boldsymbol{f} \cdot\left(\boldsymbol{v}-\boldsymbol{\Pi}_{h} \boldsymbol{v}\right)-\boldsymbol{\nabla} \boldsymbol{u}_{h}: \boldsymbol{\nabla}\left(\boldsymbol{v}-\boldsymbol{\Pi}_{h} \boldsymbol{v}\right)+p_{h} \boldsymbol{\nabla} \cdot\left(\boldsymbol{v}-\boldsymbol{\Pi}_{h} \boldsymbol{v}\right)\right\} \\
& =\int_{\Omega} \boldsymbol{f} \cdot\left(\boldsymbol{v}-\boldsymbol{\Pi}_{h} \boldsymbol{v}\right)-\sum_{K \in \mathcal{K}} \oint_{\partial K}\left(\boldsymbol{n} \cdot \boldsymbol{\nabla} \boldsymbol{u}_{h}-p_{h} \boldsymbol{n}\right) \cdot\left(\boldsymbol{v}-\boldsymbol{\Pi}_{h} \boldsymbol{v}\right) \quad \forall \boldsymbol{v} \in \boldsymbol{V},
\end{aligned}
$$

and then observe that the second term vanishes by definition of $\boldsymbol{\Pi}_{h}$ since both $\boldsymbol{n} \cdot \boldsymbol{\nabla} \boldsymbol{u}_{h}$ and $p_{h} \boldsymbol{n}$ are constant on each edge $\gamma \subset \partial K$.

3.2. Decomposition of the error. Following an idea of [12], we decompose the gradient of the error in the velocity in the form

$$
\nabla_{h} e=\nabla e_{0}+a,
$$

where $\boldsymbol{e}_{0} \in \boldsymbol{X}$ is uniquely defined by

$$
\int_{\Omega} \nabla e_{0}: \nabla v=\int_{\Omega} \nabla_{h} e: \nabla v \quad \forall v \in X
$$

The remainder $\boldsymbol{a} \in L^{2}(\Omega)^{2,2}$ satisfies

$$
\int_{\Omega} \boldsymbol{a}: \boldsymbol{\nabla v}=0 \quad \forall \boldsymbol{v} \in \boldsymbol{X}
$$

and as a consequence (3.2) defines an orthogonal splitting of the error

$$
\left\|\nabla_{h} e\right\|^{2}=\left\|\nabla e_{0}\right\|^{2}+\|\boldsymbol{a}\|^{2} .
$$

Moreover, $\boldsymbol{a}$ belongs to the closed subspace $\boldsymbol{Y}$ of $L^{2}(\Omega)^{2,2}$ defined by

$$
\boldsymbol{Y}:=\left\{\boldsymbol{w} \in L^{2}(\Omega)^{2,2}: \int_{\Omega} \boldsymbol{w}: \boldsymbol{\nabla} \boldsymbol{v}=0 \forall \boldsymbol{v} \in \boldsymbol{X}\right\} .
$$

Members of the subspace $\boldsymbol{Y}$ share the following useful property.

Lemma 2. For each $\boldsymbol{w} \in \boldsymbol{Y}$ there exists a function $\omega \in L_{0}^{2}(\Omega)$ such that

$$
\int_{\Omega} \boldsymbol{w}: \nabla \boldsymbol{v}=\int_{\Omega} \omega \boldsymbol{\nabla} \cdot \boldsymbol{v} \quad \forall \boldsymbol{v} \in \boldsymbol{V}
$$

i.e., $\boldsymbol{\nabla} \cdot \boldsymbol{w}=\boldsymbol{\nabla} \omega$, and which satisfies the estimate

$$
\|\omega\| \leq \frac{1}{c_{0}}\|\boldsymbol{w}\|
$$

where $c_{0}$ is the constant appearing in the inf-sup condition (1.3).

Proof. Integration by parts reveals that every $\boldsymbol{w} \in \boldsymbol{Y} \cap H^{1}(\Omega)^{2,2}$ satisfies

$$
-\int_{\Omega}(\boldsymbol{\nabla} \cdot \boldsymbol{w}) \cdot \boldsymbol{v}=0 \quad \forall \boldsymbol{v} \in \boldsymbol{X} .
$$

Hence, by [16, Ch. III.1], there exists $\omega \in H^{1}(\Omega) \cap L_{0}^{2}(\Omega)$ such that $\boldsymbol{\nabla} \cdot \boldsymbol{w}=\boldsymbol{\nabla} \omega$. Equation (3.6) then follows by integration by parts. The validity of the inf-sup condition means that we may pick $\boldsymbol{v} \in H_{0}^{1}(\Omega)^{2}$ such that $\boldsymbol{\nabla} \cdot \boldsymbol{v}=\omega$ and

$$
\|\omega\|^{2}=\int_{\Omega} \omega \boldsymbol{\nabla} \cdot \boldsymbol{v}=\int_{\Omega} \boldsymbol{w}: \boldsymbol{\nabla} \boldsymbol{v} \leq\|\boldsymbol{w}\|\|\boldsymbol{\nabla} \boldsymbol{v}\| \leq \frac{1}{c_{0}}\|\boldsymbol{w}\|\|\omega\|,
$$

which completes the proof of the assertion. 
Remark. More precisely, $\boldsymbol{w} \in \boldsymbol{Y}$ is of the form $\boldsymbol{w}=\omega \boldsymbol{I d}+\boldsymbol{\nabla}^{\perp} \boldsymbol{s}$ [10. since, by (3.6), we have $\boldsymbol{\nabla} \cdot(\boldsymbol{w}-\omega \boldsymbol{I d})=0$ and, therefore, $\boldsymbol{w}-\omega \boldsymbol{I d}$ must be of the form $\boldsymbol{\nabla}^{\perp} \boldsymbol{s}$ for some $s \in H^{1}(\Omega)^{2}$.

3.3. Upper a posteriori error bound. We are now in a position to describe the a posteriori error estimator and to prove that it gives computable upper bounds for the error. Let $\overline{\boldsymbol{f}}_{K}$ denote a constant approximation of $\boldsymbol{f}$ on $K$ (such as the mean value of $\boldsymbol{f}$ over $K$ ). The estimator $\eta_{\mathrm{C}}$ for the conforming part $\boldsymbol{e}_{0}$ of the error is defined by

$$
\eta_{\mathrm{C}}:=\left(\sum_{K \in \mathcal{K}} \eta_{\mathrm{C} ; K}^{2}\right)^{1 / 2} \quad \text { with } \quad \eta_{\mathrm{C} ; K}^{2}:=\frac{1}{12}|K|\left|\overline{\boldsymbol{f}}_{K}\right|^{2} \sum_{\gamma \in \overline{\mathcal{E}}: \gamma \subset \partial K}\left|\boldsymbol{x}_{\gamma}-\boldsymbol{x}_{K}\right|^{2},
$$

where, it will be recalled, $\boldsymbol{x}_{K}$ and $\boldsymbol{x}_{\gamma}$ denote the centroid of the element and an edge $\gamma$, respectively. The estimator $\eta_{\mathrm{NC}}$ for the remainder $\boldsymbol{a}$ is defined by

$$
\eta_{\mathrm{NC}}:=\left\|\nabla_{h}\left(\boldsymbol{u}^{*}-\boldsymbol{u}_{h}\right)\right\|+\frac{1}{c_{0}}\left\|\boldsymbol{\nabla} \cdot \boldsymbol{u}^{*}\right\|
$$

where $\boldsymbol{u}^{*}$ is any function in $H_{0}^{1}(\Omega)^{2}$ and $c_{0}$ is the lower bound in the inf-sup condition (1.3). The estimator for the total error $\boldsymbol{e}$ is obtained by summing the estimators

$$
\eta:=\eta_{\mathrm{C}}+\eta_{\mathrm{NC}}
$$

The presence of the constant $c_{0}$ from the inf-sup condition in the expression for $\eta_{\mathrm{NC}}$ could be removed if one were prepared to construct a divergence-free post-processed approximation $\boldsymbol{u}^{*}$. However, this is hardly a viable or practical proposition, and the presence of $c_{0}$ is essentially unavoidable. Fortunately, bounds for $c_{0}$ are available and can be obtained from [8, 25].

In order to describe the upper bound property of the estimator, it is convenient to introduce the data oscillation on $\mathcal{K}$ defined by

$$
\underset{\mathcal{K}}{\operatorname{Osc}}(\boldsymbol{f})^{2}=\sum_{K \in \mathcal{K}} \underset{K}{\operatorname{Osc}}(\boldsymbol{f})^{2}:=\sum_{K \in \mathcal{K}}|K|\left\|\boldsymbol{f}-\overline{\boldsymbol{f}}_{K}\right\|_{K}^{2} .
$$

Theorem 1 (Upper a posteriori bound). Let $\eta$ denote the estimator defined in (3.8) - (3.10). Then, for any choice $\boldsymbol{u}^{*} \in H_{0}^{1}(\Omega)^{2}$,

$$
\left\|\nabla_{h} e\right\| \leq \eta+C \underset{\mathcal{K}}{\operatorname{osc}}(\boldsymbol{f})
$$

where $C$ is a positive constant that depends only on the shape regularity of the mesh.

Proof. Thanks to the orthogonal splitting (3.5) of the error (3.2), it suffices to estimate the two contributions separately.

(i) Upper bound for $\left\|\nabla \boldsymbol{e}_{0}\right\|$. By the definition of $\boldsymbol{e}_{0}$, (3.3) and Lemma 1 we observe that for all $\boldsymbol{v} \in \boldsymbol{X}$,

$$
\begin{aligned}
\int_{\Omega} \nabla \boldsymbol{e}_{0}: \boldsymbol{\nabla} \boldsymbol{v} & =\int_{\Omega} \nabla_{h} \boldsymbol{e}: \boldsymbol{\nabla} \boldsymbol{v}=\int_{\Omega} \boldsymbol{f} \cdot\left(\boldsymbol{v}-\boldsymbol{\Pi}_{h} \boldsymbol{v}\right)=\sum_{K \in \mathcal{K}} \int_{K} \boldsymbol{f} \cdot\left(\boldsymbol{v}-\boldsymbol{\Pi}_{h} \boldsymbol{v}\right) \\
& =\sum_{K \in \mathcal{K}}\left\{\int_{K} \overline{\boldsymbol{f}}_{K} \cdot\left(\boldsymbol{v}-\boldsymbol{\Pi}_{h} \boldsymbol{v}\right)+\int_{K}\left(\boldsymbol{f}-\overline{\boldsymbol{f}}_{K}\right) \cdot\left(\boldsymbol{v}-\boldsymbol{\Pi}_{h} \boldsymbol{v}\right)\right\} \\
& =\sum_{K \in \mathcal{K}}\left\{\int_{K} \boldsymbol{\sigma}_{K}: \nabla \boldsymbol{v}+\int_{K}\left(\boldsymbol{f}-\overline{\boldsymbol{f}}_{K}\right) \cdot\left(\boldsymbol{v}-\boldsymbol{\Pi}_{h} \boldsymbol{v}\right)\right\}
\end{aligned}
$$


where $\boldsymbol{\sigma}_{K}$ is given by

$$
\boldsymbol{\sigma}_{K}(\boldsymbol{x}):=-\frac{1}{2} \overline{\boldsymbol{f}}_{K} \otimes\left(\boldsymbol{x}-\boldsymbol{x}_{K}\right) .
$$

To verify the last step, first note that

$$
\int_{K} \boldsymbol{\sigma}_{K}: \boldsymbol{\nabla} \boldsymbol{v}=\oint_{\partial K} \boldsymbol{\sigma}_{K} \boldsymbol{n}_{K} \cdot \boldsymbol{v}-\int_{K}\left(\boldsymbol{\nabla} \cdot \boldsymbol{\sigma}_{K}\right) \cdot \boldsymbol{v}
$$

Simple computations reveal that $\boldsymbol{\nabla} \cdot \boldsymbol{\sigma}_{K}=-1 / 2 \overline{\boldsymbol{f}}_{K} \boldsymbol{\nabla} \cdot\left(\boldsymbol{x}-\boldsymbol{x}_{K}\right)=-\overline{\boldsymbol{f}}_{K}$ on $K$ and that $\boldsymbol{\sigma}_{K} \boldsymbol{n}_{K}=-1 / 2 \overline{\boldsymbol{f}}_{K} \boldsymbol{n}_{K} \cdot\left(\boldsymbol{x}-\boldsymbol{x}_{K}\right)=-|K| /\left(3 h_{\gamma}\right) \overline{\boldsymbol{f}}_{K}$ on $\gamma \subset \partial K$. Hence,

$$
\oint_{\partial K} \boldsymbol{\sigma}_{K} \boldsymbol{n}_{K} \cdot \boldsymbol{v}=-\sum_{\gamma \subset \partial K} \overline{\boldsymbol{f}}_{K} \cdot \frac{1}{h_{\gamma}} \int_{\gamma} \boldsymbol{v} \frac{1}{3}|K|=-\int_{K} \overline{\boldsymbol{f}}_{K} \cdot \boldsymbol{\Pi}_{h} \boldsymbol{v}
$$

and the claim follows. Consequently, using the interpolation estimate (2.6), we obtain

$$
\left\|\boldsymbol{\nabla} \boldsymbol{e}_{0}\right\| \leq\left(\sum_{K \in \mathcal{K}}\left\|\boldsymbol{\sigma}_{K}\right\|_{K}^{2}\right)^{1 / 2}+C\left(\sum_{K \in \mathcal{K}} h_{K}^{2}\left\|\boldsymbol{f}-\overline{\boldsymbol{f}}_{K}\right\|_{K}^{2}\right)^{1 / 2}=\eta_{\mathrm{C}}+C \underset{\mathcal{K}}{\operatorname{osc}}(\boldsymbol{f}),
$$

where the last equality follows from an explicit calculation of $\left\|\boldsymbol{\sigma}_{K}\right\|_{K}^{2}$.

(ii) Upper bound for $\|\boldsymbol{a}\|$. To estimate $\boldsymbol{a}$ take any $\boldsymbol{w} \in \boldsymbol{Y}$ and choose an arbitrary element $\boldsymbol{u}^{*} \in H_{0}^{1}(\Omega)^{2}$. Then, using (3.4) and Lemma 2, we obtain

$$
\begin{aligned}
\int_{\Omega} \boldsymbol{a}: \boldsymbol{w} & =\int_{\Omega} \nabla_{h}\left(\boldsymbol{e}-\boldsymbol{e}_{0}\right): \boldsymbol{w}=\int_{\Omega} \nabla_{h}\left(-\boldsymbol{u}_{h}\right): \boldsymbol{w} \\
& =\int_{\Omega}\left\{\nabla_{h}\left(\boldsymbol{u}^{*}-\boldsymbol{u}_{h}\right): \boldsymbol{w}-\nabla \boldsymbol{u}^{*}: \boldsymbol{w}\right\} \\
& =\int_{\Omega}\left\{\nabla_{h}\left(\boldsymbol{u}^{*}-\boldsymbol{u}_{h}\right): \boldsymbol{w}-\boldsymbol{\nabla} \cdot \boldsymbol{u}^{*} \omega\right\} .
\end{aligned}
$$

The estimate now follows using the bound for $\omega$ from Lemma 2 .

3.4. Choice of $\boldsymbol{u}^{*}$. The quality of the estimator $\eta$ defined in (3.10) will depend on making a good choice for $\boldsymbol{u}^{*}$. One possibility is to construct $\boldsymbol{u}^{*}$ by post-processing the finite element approximation $\boldsymbol{u}_{h}$. With this in mind, we begin by defining a mapping $\boldsymbol{P}_{h}: \boldsymbol{X}_{h} \rightarrow \boldsymbol{V}_{h}^{*}$ onto the enhanced space $\boldsymbol{V}_{h}^{*}$ defined in Section 2.4 First, the values of the (discontinuous) function $\boldsymbol{v}_{h}$ are averaged at each node $n \in \mathcal{N}$ as follows:

$$
\boldsymbol{S}\left(\boldsymbol{v}_{h}\right)_{n}:= \begin{cases}\sum_{K \in \mathcal{K}: \boldsymbol{x}_{n} \in K} \omega_{n ; K} \boldsymbol{v}_{h ; K}\left(\boldsymbol{x}_{n}\right), & \text { for } \boldsymbol{x}_{n} \in \Omega \\ \mathbf{0}, & \text { for } \boldsymbol{x}_{n} \in \partial \Omega\end{cases}
$$

where $\left\{\omega_{n ; K}\right\}$ is any set of nonnegative weights satisfying $\sum_{K \in \mathcal{K}: \boldsymbol{x}_{n} \in K} \omega_{n ; K}=1$. Secondly, each $\boldsymbol{v}_{h} \in \boldsymbol{X}_{h}$ may be uniquely written in the form $\boldsymbol{v}_{h}=\sum_{\gamma \in \mathcal{E}} v_{\gamma} \boldsymbol{\tau}_{\gamma}+$ $\sum_{n \in \mathcal{N}} v_{n} \boldsymbol{\rho}_{n}$, and a mapping $\boldsymbol{P}_{h}: \boldsymbol{X}_{h} \rightarrow \boldsymbol{V}_{h}^{*}$ can be defined, therefore, by

$$
\boldsymbol{P}_{h} \boldsymbol{v}_{h}:=\sum_{\gamma \in \mathcal{E}} v_{\gamma} \boldsymbol{\tau}_{\gamma}^{*}+\sum_{n \in \mathcal{N}}\left\{v_{n} \boldsymbol{\rho}_{n}^{*}+\boldsymbol{S}\left(\boldsymbol{v}_{h}\right)_{n} \phi_{n}^{*}\right\}
$$

By construction, it holds that $\int_{\gamma} \boldsymbol{P}_{h} \boldsymbol{v}_{h} \cdot \boldsymbol{n}=\int_{\gamma} \boldsymbol{v}_{h} \cdot \boldsymbol{n}=0$ for all $\gamma \in \overline{\mathcal{E}}$, and it is easily verified that, for every $\boldsymbol{v}_{h} \in \boldsymbol{X}_{h}$,

$$
\int_{\Omega} \boldsymbol{\nabla} \cdot\left(\boldsymbol{P}_{h} \boldsymbol{v}_{h}\right) q_{h}=0 \quad \forall q_{h} \in M_{h}
$$


The post-processing scheme embodied in $\boldsymbol{P}_{h}$ forms the basis for a family of post-processing schemes of the form

$$
\boldsymbol{Q}_{h} \boldsymbol{v}_{h}:=\boldsymbol{P}_{h} \boldsymbol{v}_{h}+\sum_{K \in \mathcal{K}} \boldsymbol{c}_{K} \beta_{K}^{*},
$$

corresponding to various choices of the local vectors $\boldsymbol{c}_{K} \in \mathbb{R}^{2}$ for $K \in \mathcal{K}$.

The simplest choice $\boldsymbol{c}_{K}=\mathbf{0}$ gives an operator $\boldsymbol{Q}_{h}^{0}$ which coincides with the basic post-processing scheme, i.e., $\boldsymbol{Q}_{h}^{0}=\boldsymbol{P}_{h}$. Alternatively, the freedom in the choice of coefficients of the bubble functions $\beta_{K}^{*}$ may be exploited to give a post-processed approximation $\boldsymbol{Q}_{h}^{\mathrm{DDF}} \boldsymbol{v}_{h}$ belonging to the subspace $\boldsymbol{X}_{h}^{*}$ of "discretely divergencefree" functions (2.9) by taking $\boldsymbol{c}_{K} \in \mathbb{R}^{2}$ as suggested in [17]:

$$
\boldsymbol{c}_{K}^{\mathrm{DDF}}=\frac{1}{\int_{K} \beta_{K}^{*}}\left(\oint_{\partial K}\left(\boldsymbol{x}-\boldsymbol{x}_{K}\right) \boldsymbol{P}_{h} \boldsymbol{v}_{h} \cdot \boldsymbol{n}-\int_{K} \boldsymbol{P}_{h} \boldsymbol{v}_{h}\right) .
$$

Note that this choice preserves the property $\int_{\gamma} \boldsymbol{Q}_{h} \boldsymbol{u}_{h} \cdot \boldsymbol{n}=\int_{\gamma} \boldsymbol{P}_{h} \boldsymbol{u}_{h} \cdot \boldsymbol{n}=\int_{\gamma} \boldsymbol{u}_{h} \cdot \boldsymbol{n}$ for all $\gamma \in \overline{\mathcal{E}}$. The construction of the discretely divergence-free post-processing scheme $\boldsymbol{Q}_{h}^{\mathrm{DDF}}$ is motivated by the desire to implicitly control the influence of the term in (3.9) involving the inf-sup constant $c_{0}$. A more direct approach is to choose the constants $\boldsymbol{c}_{K}$ to minimize this term. This can be achieved by choosing

$$
\int_{K} \boldsymbol{\nabla} \beta_{K}^{*} \otimes \boldsymbol{\nabla} \beta_{K}^{*} \boldsymbol{c}_{K}^{\mathrm{MIN}}=-\int_{K} \boldsymbol{\nabla} \cdot\left(\boldsymbol{P}_{h} \boldsymbol{u}_{h}\right) \boldsymbol{\nabla} \beta_{K}^{*},
$$

and the associated "minimal-divergence" post-processing scheme is denoted by $\boldsymbol{Q}_{h}^{\mathrm{MIN}}$. Finally, in view of the fact that one obtains an upper bound regardless of the choice of $\boldsymbol{c}_{K}$, we consider the post-processing scheme whereby the coefficients $\boldsymbol{c}_{K}$ are chosen to minimize the upper bound:

$$
\begin{aligned}
& \left(\left\|\boldsymbol{\nabla} \beta_{K}^{*}\right\|_{K}^{2} \boldsymbol{I} \boldsymbol{d}+\frac{1}{c_{0}^{2}} \int_{K} \boldsymbol{\nabla} \beta_{K}^{*} \otimes \boldsymbol{\nabla} \beta_{K}^{*}\right) \boldsymbol{c}_{K}^{\mathrm{OPT}} \\
& \quad=-\int_{K}\left(\boldsymbol{\nabla} \boldsymbol{P}_{h} \boldsymbol{u}_{h}+\frac{1}{c_{0}^{2}} \boldsymbol{\nabla} \cdot\left(\boldsymbol{P}_{h} \boldsymbol{u}_{h}\right) \boldsymbol{I} \boldsymbol{d}\right) \boldsymbol{\nabla} \beta_{K}^{*},
\end{aligned}
$$

and we denote the associated post-processing operator by $\boldsymbol{Q}_{h}^{\mathrm{OPT}}$.

3.5. Lower a posteriori error bound. Each of the post-processing schemes defined in the previous section gives $\boldsymbol{Q}_{h} \boldsymbol{u}_{\boldsymbol{h}} \in H_{0}^{1}(\Omega)^{2}$ and, therefore, we may select $\boldsymbol{u}^{*}=\boldsymbol{Q}_{h} \boldsymbol{u}_{\boldsymbol{h}}$ in Theorem 1 to obtain upper bounds on the error. We shall show that each of these upper bounds also provides an efficient bound provided that the triangulation satisfies the additional condition whereby each element $K \in \mathcal{K}$ should have at most one edge on the exterior boundary $\partial \Omega$. Obviously, this assumption is not a serious practical limitation since it may be satisfied by performing a suitable refinement of any triangles that do not meet this requirement.

Theorem 2 (Lower a posteriori bound). Suppose that each element $K \in \mathcal{K}$ has at most one edge on the exterior boundary. If the estimator $\eta_{\mathrm{NC}}$ is defined by taking $\boldsymbol{u}^{*}=\boldsymbol{Q}_{h} \boldsymbol{u}_{h}$, where $\boldsymbol{Q}_{h}$ is any of the operators defined in Section [3.4, then

$$
c \eta \leq\left\|\nabla_{h} e\right\|+\underset{\mathcal{K}}{\operatorname{osc}}(\boldsymbol{f}),
$$

where $c$ is a positive constant that depends only on the shape regularity of the mesh. 
Proof. The orthogonality of the splitting (3.2) means that it suffices to prove lower bounds for $\left\|\nabla e_{0}\right\|$ and $\|\boldsymbol{a}\|$ individually.

(i) Lower bound for $\left\|\nabla e_{0}\right\|$. Let $\bar{f}$ denote the piecewise constant function whose value on $K$ is given by $\overline{\boldsymbol{f}}_{K}$, and let $\boldsymbol{\sigma}$ be defined as in the proof of Theorem 1 . Then, for all $\boldsymbol{v} \in \boldsymbol{X}$, we have

$$
\int_{\Omega} \nabla e_{0}: \nabla \boldsymbol{v}=\int_{\Omega} \boldsymbol{\sigma}: \nabla \boldsymbol{v}+\int_{\Omega}(f-\bar{f}) \cdot\left(\boldsymbol{v}-\boldsymbol{\Pi}_{h} \boldsymbol{v}\right) .
$$

Let $K^{\prime}, K^{\prime \prime} \in \mathcal{K}$ be distinct elements sharing the common edge $\gamma=K^{\prime} \cap K^{\prime \prime}$, and denote $\Omega_{\gamma}:=K^{\prime} \cup K^{\prime \prime}$. Let $s \in C_{0}^{2}\left(\Omega_{\gamma}\right)$ be compactly supported in $\Omega_{\gamma}$, and set $v:=\nabla^{\perp} s$. Then, with the aid of (2.6), we obtain

$$
\frac{1}{\|\boldsymbol{\nabla} \boldsymbol{v}\|}\left|\int_{\Omega_{\gamma}} \boldsymbol{\sigma}: \boldsymbol{\nabla} \boldsymbol{v}\right| \leq\left\|\boldsymbol{\nabla} \boldsymbol{e}_{0}\right\|_{\Omega_{\gamma}}+C \underset{\Omega_{\gamma}}{\operatorname{osc}}(\boldsymbol{f}) .
$$

Moreover, from the definition of $\boldsymbol{\sigma}$,

$$
\begin{aligned}
\int_{\Omega} \boldsymbol{\sigma}: \boldsymbol{\nabla} \boldsymbol{v} & =\sum_{K \in\left\{K^{\prime}, K^{\prime \prime}\right\}} \int_{K} \overline{\boldsymbol{f}}_{K} \cdot\left(\boldsymbol{v}-\boldsymbol{\Pi}_{h} \boldsymbol{v}\right) \\
& =\sum_{K \in\left\{K^{\prime}, K^{\prime \prime}\right\}}\left\{-\frac{|K|}{3 h_{\gamma}} \overline{\boldsymbol{f}}_{K} \cdot \int_{\gamma} \boldsymbol{\nabla}^{\perp} s+\overline{\boldsymbol{f}}_{K} \cdot \boldsymbol{t}_{K} \int_{\gamma} s\right\} .
\end{aligned}
$$

Observe that $\boldsymbol{t}_{K^{\prime}}+\boldsymbol{t}_{K^{\prime \prime}}=\mathbf{0}$ on $\gamma$, and so, for the sake of definiteness, we fix $\boldsymbol{t}_{K^{\prime}}=\boldsymbol{t}_{\gamma}$. Furthermore,

$$
\int_{\gamma} \boldsymbol{\nabla}^{\perp} s=\int_{\gamma}\left(\boldsymbol{n}_{\gamma} \cdot \boldsymbol{\nabla}^{\perp} s\right) \boldsymbol{n}_{\gamma}+\int_{\gamma}\left(\boldsymbol{t}_{\gamma} \cdot \boldsymbol{\nabla}^{\perp} s\right) \boldsymbol{t}_{\gamma}=\int_{\gamma} \partial_{\boldsymbol{n}} s \boldsymbol{t}_{\gamma},
$$

where the first integral vanishes since $s$ vanishes at the endpoints of $\gamma$. Hence, setting $\lambda_{0} \equiv \lambda_{0}(s):=f_{\gamma} s$ and $\lambda_{1} \equiv \lambda_{1}(s):=\int_{\gamma} \partial_{\boldsymbol{n}} s$, we obtain

$$
\frac{1}{h_{\gamma}} \int_{\Omega_{\gamma}} \boldsymbol{\sigma}: \nabla \boldsymbol{v}=-\frac{1}{3}\left(\frac{\left|K^{\prime}\right|}{h_{\gamma}^{2}} \overline{\boldsymbol{f}}_{K^{\prime}}+\frac{\left|K^{\prime \prime}\right|}{h_{\gamma}^{2}} \overline{\boldsymbol{f}}_{K^{\prime \prime}}\right) \cdot \boldsymbol{t}_{\gamma} \lambda_{1}+\left(\overline{\boldsymbol{f}}_{K^{\prime}}-\overline{\boldsymbol{f}}_{K^{\prime \prime}}\right) \cdot \boldsymbol{t}_{\gamma} \lambda_{0} .
$$

Observe that the right hand side is invariant under a rescaling of the domain and so without loss of generality we may assume that $h_{\gamma}=1$. The main idea is, through appropriate choices of $s$, to show that (3.17) gives rise to a pair of linearly independent equations in which the tangential components of $\boldsymbol{f}_{K^{\prime}}$ and $\boldsymbol{f}_{K^{\prime \prime}}$ are regarded as unknowns. The choices of $s$ are constructed as follows. First, let $\varrho$ be the largest value such that $B_{\varrho}\left(\boldsymbol{x}_{\gamma}\right) \subset \Omega_{\gamma}$. Let $s$ be a nonnegative function with support $\operatorname{supp}(s)=B_{\varrho / 2}\left(\boldsymbol{x}_{\gamma}\right)$ and such that $s$ is radially symmetric about the midpoint $\boldsymbol{x}_{\gamma}$, normalized so that $\max (s)=1$. Let $\delta$ satisfy $0 \leq \delta<\varrho / 2$ and consider the functions $s_{\delta}:=s\left(.-\delta \boldsymbol{n}_{\gamma}\right)$ obtained by shifting the support of $s$. The mapping $\delta \mapsto \lambda_{0}\left(s_{\delta}\right)$ is then a positive, symmetric and strictly decreasing function and $\delta \mapsto \lambda_{1}\left(s_{\delta}\right)$ is a nonconstant skew-symmetric function. Taking first $\delta=0$ and then $\delta=\delta_{0}$ for some fixed value $\delta_{0} \in(0, \varrho / 2)$, we find that (3.17) gives a pair of linearly independent equations for the tangential components of $\boldsymbol{f}_{K^{\prime}}$ and $\boldsymbol{f}_{K^{\prime \prime}}$. Consequently, there is a constant $C$, depending only on the shape of $\Omega_{\gamma}$, such that

$$
\left|\overline{\boldsymbol{f}}_{K^{\prime}} \cdot \boldsymbol{t}_{\gamma}\right| \leq \frac{C}{h_{\gamma}}\left|\int_{\Omega_{\gamma}} \boldsymbol{\sigma}: \boldsymbol{\nabla} \boldsymbol{v}\right|,
$$


where we have exploited the fact that $\|\boldsymbol{\nabla} \boldsymbol{v}\|_{\Omega_{\gamma}}$ is independent of $\delta$. In fact, by a scaling argument, we deduce that there is a positive constant $C$, again independent of $\delta$, such that $\|\boldsymbol{\nabla} \boldsymbol{v}\|_{\Omega_{\gamma}} \leq C / h_{\gamma}$. Hence, with (3.16) and (3.18) we get

$$
\left|K^{\prime}\right|^{1 / 2}\left\|\overline{\boldsymbol{f}}_{K^{\prime}} \cdot \boldsymbol{t}_{\gamma}\right\|_{K^{\prime}} \leq C h_{\gamma}^{2}\left|\overline{\boldsymbol{f}}_{K^{\prime}} \cdot \boldsymbol{t}_{\gamma}\right| \leq C\left(\left\|\boldsymbol{\nabla} \boldsymbol{e}_{0}\right\|_{\Omega_{\gamma}}+\underset{\Omega_{\gamma}}{\operatorname{osc}}(\boldsymbol{f})\right) .
$$

Since each element is assumed to have at most one edge on the exterior boundary, we can apply the same reasoning to another edge $\gamma \neq \gamma^{\prime}$ contained in $\partial K^{\prime}$. Further, since the set $\left\{\boldsymbol{t}_{\gamma}, \boldsymbol{t}_{\gamma^{\prime}}\right\}$ is linearly independent, we are able to estimate both components of $\boldsymbol{f}_{K^{\prime}}$ and thereby arrive at the bound

$$
\left|K^{\prime}\right|^{1 / 2}\left\|\overline{\boldsymbol{f}}_{K^{\prime}}\right\|_{K^{\prime}} \leq C\left(\left\|\nabla \boldsymbol{e}_{0}\right\|_{\Omega_{\gamma} \cup \Omega_{\gamma^{\prime}}}+\underset{\Omega_{\gamma} \cup \Omega_{\gamma^{\prime}}}{\operatorname{osc}}(\boldsymbol{f})\right) .
$$

Summation over all elements $K \in \mathcal{K}$ then yields

$$
c \eta \leq\left\|\nabla e_{0}\right\|+\underset{\mathcal{K}}{\operatorname{osc}}(\boldsymbol{f}),
$$

since $\eta_{\mathrm{C} ; K} \leq C\left|\overline{\boldsymbol{f}}_{K}\right|\left\|\boldsymbol{x}-\boldsymbol{x}_{K}\right\|_{K} \leq C\left|\overline{\boldsymbol{f}}_{K}\right||K| \leq C|K|^{1 / 2}\left\|\overline{\boldsymbol{f}}_{K}\right\|_{K}$.

(ii) Lower bound for $\|\boldsymbol{a}\|$. The proof consists of two steps. In the first step we show that $\|\boldsymbol{a}\|$ bounds a sum of jumps $J_{\boldsymbol{t}}$ (defined below) in the tangential derivatives of $\boldsymbol{u}_{h}$. Then, in the second step we show that these jumps bound the error estimator above.

(ii.1) $\|\boldsymbol{a}\|$ bounds $J_{\boldsymbol{t}}$. Let $K^{\prime}$ and $K^{\prime \prime}$ be distinct elements sharing a common edge $\gamma=K^{\prime} \cap K^{\prime \prime}$ and let $s:=\theta_{\gamma}^{*} \boldsymbol{z}_{\gamma}$, where $\theta_{\gamma}^{*}$ is defined as in Section 2.4 and $\boldsymbol{z}_{\gamma} \in \mathbb{R}^{2}$ is a constant to be specified later. The function $\nabla^{\perp} s$ is divergence-free and therefore $\nabla^{\perp} s \in \boldsymbol{Y}$. Then, arguing as in the proof of Theorem 1(ii), we deduce that

$$
\begin{aligned}
\int_{\Omega} \boldsymbol{a}: \nabla^{\perp} \boldsymbol{s} & =-\int_{K^{\prime} \cup K^{\prime \prime}} \nabla_{h} \boldsymbol{u}_{h}: \nabla^{\perp} \boldsymbol{s}=-\sum_{K \in\left\{K^{\prime}, K^{\prime \prime}\right\}} \oint_{\partial K}\left(\boldsymbol{t}_{K} \cdot \boldsymbol{\nabla} \boldsymbol{u}_{h}\right) \cdot \boldsymbol{s} \\
& =-\int_{\gamma}\left[\partial_{\boldsymbol{t}} \boldsymbol{u}_{h}\right]_{\gamma} \cdot \boldsymbol{s} .
\end{aligned}
$$

Choose $\boldsymbol{z}_{\gamma}:=-\left[\partial_{\boldsymbol{t}} \boldsymbol{u}_{h}\right]_{\gamma}$. Then

$$
\begin{aligned}
J_{\boldsymbol{t} ; \gamma}^{2} & :=h_{\gamma} \int_{\gamma}\left|\left[\partial_{\boldsymbol{t}} \boldsymbol{u}_{h}\right]_{\gamma}\right|^{2}=h_{\gamma} \int_{\gamma} \theta_{\gamma}^{*}\left|\left[\partial_{\boldsymbol{t}} \boldsymbol{u}_{h}\right]_{\gamma}\right|^{2}=-h_{\gamma} \int_{K^{\prime} \cup K^{\prime \prime}} \boldsymbol{a}: \boldsymbol{\nabla}^{\perp}\left(\theta_{\gamma}^{*}\left[\partial_{\boldsymbol{t}} \boldsymbol{u}_{h}\right]_{\gamma}\right) \\
& \leq\|\boldsymbol{a}\|_{K^{\prime} \cup K^{\prime \prime}}\left\|\boldsymbol{\nabla}^{\perp} \theta_{\gamma}^{*}\right\| J_{\boldsymbol{t} ; \gamma},
\end{aligned}
$$

and by summing over $\gamma \in \mathcal{E}$ we obtain

$$
J_{\boldsymbol{t}}:=\left(\sum_{\gamma \in \mathcal{E}} J_{\boldsymbol{t} ; \gamma}^{2}\right)^{1 / 2} \leq C\|\boldsymbol{a}\|,
$$

where $C$ is a positive constant independent of any mesh-size.

(ii.2) $J_{\boldsymbol{t}}$ bounds $\eta_{\mathrm{NC}}$. To this end take any $K \in \mathcal{K}$ and let $\mathcal{A}_{K}:=\left\{\gamma^{\prime} \in \mathcal{E}: \gamma^{\prime} \subset\right.$ $\left.\Omega_{K}\right\}$ be the set of all edges in $\Omega_{K}:=\bigcup\left\{K^{\prime} \in \mathcal{K}: K \cap K^{\prime} \neq \emptyset\right\}$. We now wish to bound

$$
q_{K}:=\frac{\left\|\nabla\left(\boldsymbol{u}^{*}-\boldsymbol{u}_{h}\right)\right\|_{K}^{2}}{\sum_{\gamma \in \mathcal{A}_{K}} J_{\boldsymbol{t} ; \gamma}^{2}}
$$

from above. To finish, observe that $\eta_{\mathrm{NC}} \leq C\left\|\nabla_{h}\left(\boldsymbol{u}^{*}-\boldsymbol{u}_{h}\right)\right\|$. The form of $\boldsymbol{u}^{*}$ means that the denominator depends on an at least as large a set of coefficients of $\boldsymbol{u}_{h}$ as the numerator. Since $q_{K}$ is the quotient of two homogeneous quadratic forms, 
it suffices to show that if the denominator vanishes, then so does the numerator. Thanks to a scaling argument, we may eliminate the mesh-size and consider a patch $\Omega_{K}$ of elements of unit diameter. Suppose now that $J_{\boldsymbol{t} ; \gamma}=0$ holds for all $\gamma \in \mathcal{A}_{K}$. If $\boldsymbol{x}_{n}$ is any vertex of $K$ and $\gamma=K \cap K^{\prime}$, then by continuity in $\boldsymbol{x}_{\gamma}$ we have $\left|\boldsymbol{u}_{h ; K}\left(\boldsymbol{x}_{n}\right)-\boldsymbol{u}_{h ; K^{\prime}}\left(\boldsymbol{x}_{n}\right)\right| \leq \int_{\gamma}\left|\left[\partial_{\boldsymbol{t}} \boldsymbol{u}_{h}\right]\right|=0$. Because all edges emanating from $\boldsymbol{x}_{n}$ are in $\mathcal{A}_{K}$, we conclude that $\boldsymbol{u}_{h}$ is continuous at $\boldsymbol{x}_{n}$. Since it is linear, it is thus continuous in $\Omega_{K}$ and $\boldsymbol{u}^{*}=\boldsymbol{u}_{h}$ on $\partial K$. Thus $\boldsymbol{u}_{h ; K}=\boldsymbol{P}_{h} \boldsymbol{u}_{h \bigsqcup_{K}}$ and therefore $\boldsymbol{u}^{*}=\boldsymbol{u}_{h}+\boldsymbol{d}_{K} \beta_{K}^{*}$ on $K$. If we show that $\boldsymbol{d}_{K}=\mathbf{0}$ holds, we have that $q_{K}$ is bounded by an $h_{K}$-independent constant, and we arrive at the bound $\left\|\nabla_{h}\left(\boldsymbol{u}^{*}-\boldsymbol{u}_{h}\right)\right\| \leq C J_{\boldsymbol{t}}$ after summation over $K$. By definition $\boldsymbol{d}_{K}$ vanishes in the case where $\boldsymbol{u}^{*}$ is defined using $\boldsymbol{Q}_{h}^{0}$. In the case of $\boldsymbol{Q}_{h}^{\mathrm{DDF}}$, the fact that both $\boldsymbol{u}^{*}$ and $\boldsymbol{u}_{h}$ are solenoidal implies that $\boldsymbol{d}_{K}$ again vanishes. Likewise, since $\boldsymbol{Q}_{h}^{\mathrm{MIN}}$ is chosen to minimize the difference in the divergences of $\boldsymbol{u}^{*}$ and $\boldsymbol{u}_{h}$, we conclude that $\boldsymbol{d}_{K}$ vanishes in this case also, and a similar argument applies in the case of $\boldsymbol{Q}_{h}^{\mathrm{OPT}}$.

We conclude this section by stating the a posteriori estimate for the combined error in velocity and pressure.

Corollary 1 (A posteriori error estimate for the pressure). If the previous conditions and assumptions hold, then the error in the pressure is bounded above as follows:

$$
c_{0}\left\|e_{p}\right\| \leq 2 \eta+C \underset{\mathcal{K}}{\operatorname{osc}}(\boldsymbol{f})
$$

Therefore, the total error $\left\|\nabla_{h} \boldsymbol{e}\right\|+c_{0}\left\|e_{p}\right\|$ is equivalent to our error estimate up to data oscillation terms.

Proof. First, applying Theorem 2, we at once derive

$$
c \eta \leq\left\|\nabla_{h} \boldsymbol{e}\right\|+\underset{\mathcal{K}}{\operatorname{osc}}(\boldsymbol{f}) \leq\left\|\nabla_{h} \boldsymbol{e}\right\|+c_{0}\left\|e_{p}\right\|+\underset{\mathcal{K}}{\operatorname{osc}}(\boldsymbol{f}) .
$$

It remains to give the upper a posteriori bound for $\left\|e_{p}\right\|$. To this end, notice that thanks to the inf-sup condition (1.3),

$$
c_{0}\left\|e_{p}\right\| \leq \sup _{\boldsymbol{v} \in \boldsymbol{V} \backslash\{\mathbf{0}\}}\left\{\frac{1}{\|\boldsymbol{\nabla} \boldsymbol{v}\|} \int_{\Omega} e_{p} \boldsymbol{\nabla} \cdot \boldsymbol{v}\right\} .
$$

From the error representation formula in Lemma 1 we obtain

$$
\begin{aligned}
\frac{1}{\|\boldsymbol{\nabla} \boldsymbol{v}\|} \int_{\Omega} e_{p} \boldsymbol{\nabla} \cdot \boldsymbol{v} & =\frac{1}{\|\boldsymbol{\nabla} \boldsymbol{v}\|} \int_{\Omega}\left\{\boldsymbol{\nabla}_{h} \boldsymbol{e}: \boldsymbol{\nabla} \boldsymbol{v}+\boldsymbol{f} \cdot\left(\boldsymbol{v}-\boldsymbol{\Pi}_{h} \boldsymbol{v}\right)\right\} \\
& \leq\left\|\boldsymbol{\nabla}_{h} \boldsymbol{e}\right\|+\left(\sum_{K \in \mathcal{K}}\left\|\boldsymbol{\sigma}_{K}\right\|_{K}^{2}\right)^{1 / 2}+C \underset{\mathcal{K}}{\operatorname{osc}}(\boldsymbol{f}) \leq 2 \eta+C \underset{\mathcal{K}}{\operatorname{osc}}(\boldsymbol{f}),
\end{aligned}
$$

where we used arguments as in the proof of Theorem 1(i). Hence

$$
\left\|\nabla_{h} \boldsymbol{e}\right\|+c_{0}\left\|e_{p}\right\| \leq 3 \eta+C \underset{\mathcal{K}}{\operatorname{osc}}(\boldsymbol{f})
$$

as claimed. 


\section{EXTENSION TO NONHOMOGENEOUS BOUNDARY DATA}

Consider the case of nonhomogeneous boundary conditions in which problem (1.1) is modified by requiring $\boldsymbol{u}=\boldsymbol{u}^{\partial}$ on $\partial \Omega$ for some $\boldsymbol{u}^{\partial} \in H^{1}(\partial \Omega)^{2}$ satisfying $\oint_{\partial \Omega} \boldsymbol{u}^{\partial} \cdot \boldsymbol{n}=0$. The latter condition is necessary for the problem to be well-posed. Further details concerning existence theory in this situation may be found in [16. Ch. IV]. Here, we will restrict ourselves to outlining the modifications necessary to extend our previous results, Theorem 1 and Theorem 2 to cover this situation. The nonhomogeneous boundary conditions are applied to the discrete problem by requiring that

$$
\int_{\gamma} \boldsymbol{u}_{h}=\int_{\gamma} \boldsymbol{u}^{\partial} \quad \forall \gamma \in \mathcal{E}^{\partial}
$$

Note that applying the boundary conditions in this fashion guarantees that $\oint_{\partial \Omega} \boldsymbol{u}_{h}$. $\boldsymbol{n}=0$. The enhanced approximation $\boldsymbol{Q}_{h} \boldsymbol{u}_{h}$ from Section 2 is modified to satisfy the condition $\boldsymbol{Q}_{h} \boldsymbol{u}_{h}\left(\boldsymbol{x}_{n}\right):=\boldsymbol{u}^{\partial}\left(\boldsymbol{x}_{n}\right)$ for all $\boldsymbol{x}_{n} \in \partial \Omega$. In particular, there holds $\int_{\gamma} \boldsymbol{Q}_{h} \boldsymbol{u}_{h} \cdot \boldsymbol{n}=\int_{\gamma} \boldsymbol{u}_{h} \cdot \boldsymbol{n}=\int_{\gamma} \boldsymbol{u}^{\partial} \cdot \boldsymbol{n}$ for all $\gamma \in \mathcal{E}^{\partial}$. For given data $\boldsymbol{u}^{\partial} \in H^{1}(\partial \Omega)^{2}$, the theoretical bounds will involve the quantity

$$
\underset{\mathcal{E}^{\partial}}{\operatorname{Osc}}\left(\partial_{\boldsymbol{t}} \boldsymbol{u}^{\partial}\right)^{2}=\sum_{\gamma \in \mathcal{E}^{\partial}} \underset{\gamma}{\operatorname{osc}}\left(\partial_{\boldsymbol{t}} \boldsymbol{u}^{\partial}\right)^{2}:=\sum_{\gamma \in \mathcal{E}^{\partial}} h_{\gamma}\left\|\partial_{\boldsymbol{t}} \boldsymbol{u}^{\partial}-\left(\overline{\partial_{\boldsymbol{t}} \boldsymbol{u}^{\partial}}\right)_{\gamma}\right\|_{\gamma}^{2},
$$

where $\overline{(.)}_{\gamma}$ denotes the mean value integral over $\gamma$.

Theorem 3 (A posteriori bounds in case of nonhomogeneous boundary conditions). Suppose that the estimator $\eta_{\mathrm{NC}}$ is obtained by choosing $\boldsymbol{u}^{*}:=\boldsymbol{Q}_{h} \boldsymbol{u}_{h}$. Then, Theorem 1 is modified to

$$
\left\|\nabla_{h} e\right\| \leq \eta+C\left(\underset{\mathcal{K}}{\operatorname{Osc}}(\boldsymbol{f})+\underset{\mathcal{E}^{\partial}}{\operatorname{osc}}\left(\partial_{\boldsymbol{t}} \boldsymbol{u}^{\partial}\right)\right)
$$

while Theorem 2 is modified to

$$
c \eta \leq\left\|\nabla_{h} \boldsymbol{e}\right\|+\underset{\mathcal{K}}{\operatorname{osc}}(\boldsymbol{f})+\underset{\mathcal{E}^{\partial}}{\operatorname{osc}}\left(\partial_{\boldsymbol{t}} \boldsymbol{u}^{\partial}\right) .
$$

The constants c, $C$ depend only on the shape regularity of the mesh.

Proof. We outline the necessary modifications to the proofs given earlier in the case of homogeneous data. The splitting of the error remains as stated in (3.2), but the arguments in the proofs of Theorems 1 and 2 have to be modified when we come to bound $\|\boldsymbol{a}\|$. To extend our previous results to nonhomogeneous data, it is useful to introduce a function $\boldsymbol{v}^{*} \in H^{1}(\Omega)^{2}$ with exact boundary values $\boldsymbol{u}^{\partial}$. On an interior element $K \in \mathcal{K}$, we simply take $\boldsymbol{v}^{*}:=\boldsymbol{u}^{*}$. A more elaborate argument is needed for a triangle $K$ with an exterior edge $\gamma$. First, define $\boldsymbol{\xi}_{K}^{\partial}:=\mathbf{0}$ on $\partial K \backslash \gamma$ and $\boldsymbol{\xi}_{K}^{\partial}:=\boldsymbol{u}^{\partial}-\boldsymbol{u}^{*}$ on $\gamma$. By construction $\oint_{\partial K} \boldsymbol{\xi}_{K}^{\partial} \cdot \boldsymbol{n}=\int_{\gamma}\left(\boldsymbol{u}^{\partial}-\boldsymbol{u}^{*}\right) \cdot \boldsymbol{n}=0$. Hence, an elementwise Stokes problem on $K$ with boundary data given by $\boldsymbol{\xi}_{K}^{\partial}$ has a solution that we denote by $\boldsymbol{\xi}_{K}$ and which satisfies the bound $\left\|\boldsymbol{\nabla} \boldsymbol{\xi}_{K}\right\|_{K} \leq C \operatorname{osc}_{\gamma}\left(\partial_{\boldsymbol{t}} \boldsymbol{u}^{\partial}\right)$ (obtained using arguments similar to those of [2, Lemma 8]). On each such element $K$ we define $\boldsymbol{v}^{*}:=\boldsymbol{u}^{*}+\boldsymbol{\xi}_{K}$. In summary, the function $\boldsymbol{v}^{*} \in H^{1}(\Omega)^{2}$ satisfies $\boldsymbol{v}^{*}=\boldsymbol{u}^{\partial}$ on $\partial \Omega$. 
(i) Upper bound for $\|\boldsymbol{a}\|$. These previous definitions mean that step (ii) in the proof of Theorem 1 has to be modified as

$$
\begin{aligned}
\int_{\Omega} \boldsymbol{a}: \boldsymbol{w} & =\int_{\Omega} \nabla_{h}\left(\boldsymbol{e}-\boldsymbol{e}_{0}\right): \boldsymbol{w}=\int_{\Omega} \nabla_{h}\left(\boldsymbol{u}-\boldsymbol{u}_{h}\right): \boldsymbol{w} \\
& =\int_{\Omega}\left\{\nabla_{h}\left(\boldsymbol{v}^{*}-\boldsymbol{u}_{h}\right): \boldsymbol{w}+\nabla\left(\boldsymbol{u}-\boldsymbol{v}^{*}\right): \boldsymbol{w}\right\} \\
& =\int_{\Omega}\left\{\nabla_{h}\left(\boldsymbol{v}^{*}-\boldsymbol{u}_{h}\right): \boldsymbol{w}-\boldsymbol{\nabla} \cdot \boldsymbol{u}^{*} \omega\right\} \\
& =\int_{\Omega}\left\{\nabla_{h}\left(\boldsymbol{u}^{*}-\boldsymbol{u}_{h}\right): \boldsymbol{w}-\boldsymbol{\nabla} \cdot \boldsymbol{u}^{*} \omega\right\}+\sum_{K \in \mathcal{K}} \int_{K} \nabla \boldsymbol{\xi}_{K}: \boldsymbol{w}
\end{aligned}
$$

since $\boldsymbol{\nabla} \cdot \boldsymbol{\xi}_{K}=0$. It only remains to estimate the final term:

$$
\sum_{K \in \mathcal{K}} \int_{K} \nabla \boldsymbol{\xi}_{K}: \boldsymbol{w} \leq\left(\sum_{K \in \mathcal{K}}\left\|\nabla \boldsymbol{\xi}_{K}\right\|_{K}^{2}\right)^{1 / 2}\|\boldsymbol{w}\| \leq C \underset{\mathcal{E}^{\partial}}{\operatorname{Osc}}\left(\partial_{\boldsymbol{t}} \boldsymbol{u}^{\partial}\right)\|\boldsymbol{w}\| .
$$

(ii) Lower bound for $\|\boldsymbol{a}\|$. We only have to reconsider part (ii.2) of the proof to Theorem 2. We will split $\boldsymbol{u}^{*}-\boldsymbol{u}_{h}=\left(\boldsymbol{u}^{*}-\widetilde{\boldsymbol{u}}^{*}\right)+\left(\widetilde{\boldsymbol{u}}^{*}-\boldsymbol{u}_{h}\right)$, where $\widetilde{\boldsymbol{u}}^{*}$ is as $\boldsymbol{u}^{*}$, but with $\widetilde{\boldsymbol{u}}^{*}\left(\boldsymbol{x}_{n}\right):=\boldsymbol{S}\left(\boldsymbol{u}_{h}\right)_{n}$ also for $\boldsymbol{x}_{n} \in \mathcal{N}^{\partial}$ in contrast to $\boldsymbol{u}^{*}\left(\boldsymbol{x}_{n}\right)=\boldsymbol{u}^{\partial}\left(\boldsymbol{x}_{n}\right)$. The term $\left\|\boldsymbol{\nabla}\left(\widetilde{\boldsymbol{u}}^{*}-\boldsymbol{u}_{h}\right)\right\|$ is treated as in part (ii) before, so that it remains to bound $\left\|\nabla\left(\boldsymbol{u}^{*}-\widetilde{\boldsymbol{u}}^{*}\right)\right\|$

First consider an element $K \in \mathcal{K}$ with a boundary edge $\gamma$ and boundary vertices $\boldsymbol{x}_{n}, \boldsymbol{x}_{m}$. The following argument is based on the observation

$$
\left\|\boldsymbol{\nabla}\left(\boldsymbol{u}^{*}-\widetilde{\boldsymbol{u}}^{*}\right)\right\|_{K} \leq C \max _{l \in\{n, m\}}\left|\left(\boldsymbol{u}_{h ; K}-\boldsymbol{u}^{\partial}\right)\left(\boldsymbol{x}_{l}\right)\right| .
$$

Using the discrete boundary conditions yields $\left(\boldsymbol{u}_{h ; K}-\boldsymbol{u}^{\partial}\right)\left(\boldsymbol{x}_{n}\right)=h_{\gamma} / 2 \partial_{\boldsymbol{t}} \boldsymbol{u}_{h ; K}+$ $f_{\gamma} \boldsymbol{u}^{\partial}-\boldsymbol{u}^{\partial}\left(\boldsymbol{x}_{n}\right)$, and with arguments as in the proof of Theorem 2(i.1) with $s:=$ $\theta_{\gamma}^{*} z_{\gamma}$

$$
\begin{aligned}
\int_{K} \boldsymbol{a}: \nabla^{\perp} \boldsymbol{s} & =\left(\int_{\gamma} \theta_{\gamma}^{*} \partial_{\boldsymbol{t}} \boldsymbol{u}^{\partial}-h_{\gamma} \partial_{\boldsymbol{t}} \boldsymbol{u}_{h}\right) \cdot \boldsymbol{z}_{\gamma} \\
& =\left(-2\left(\boldsymbol{u}_{h ; K}-\boldsymbol{u}^{\partial}\right)\left(\boldsymbol{x}_{n}\right)+\int_{\gamma} \theta_{\gamma}^{*} \partial_{\boldsymbol{t}} \boldsymbol{u}^{\partial}+2 f_{\gamma} \boldsymbol{u}^{\partial}-2 \boldsymbol{u}^{\partial}\left(\boldsymbol{x}_{n}\right)\right) \cdot \boldsymbol{z}_{\gamma}
\end{aligned}
$$

or

$$
\begin{aligned}
& 2\left(\boldsymbol{u}_{h ; K}-\boldsymbol{u}^{\partial}\right)\left(\boldsymbol{x}_{n}\right) \cdot \boldsymbol{z}_{\gamma} \\
&=-\int_{K} \boldsymbol{a}: \boldsymbol{\nabla}^{\perp} \boldsymbol{s}+\left(\int_{\gamma} \theta_{\gamma}^{*}\left(\partial_{\boldsymbol{t}} \boldsymbol{u}^{\partial}-\left(\overline{\partial_{\boldsymbol{t}} \boldsymbol{u}^{\partial}}\right)_{\gamma}\right)\right. \\
&\left.\quad+2 f_{\gamma} \boldsymbol{u}^{\partial}-\left(\boldsymbol{u}^{\partial}\left(\boldsymbol{x}_{n}\right)+\boldsymbol{u}^{\partial}\left(\boldsymbol{x}_{m}\right)\right)\right) \cdot \boldsymbol{z}_{\gamma} .
\end{aligned}
$$

The last two terms can be written in the form $\int_{\gamma} \chi_{\gamma}\left(\partial_{\boldsymbol{t}} \boldsymbol{u}^{\partial}-\left(\overline{\partial_{\boldsymbol{t}} \boldsymbol{u}^{\partial}}\right)_{\gamma}\right)$ with $\left|\chi_{\gamma}\right|=1 / 2$ (see [2, Lemma 9]) so we conclude, choosing $\boldsymbol{z}_{\gamma}=[1 ; 0]$ and $[0 ; 1]$, that

$$
\left|\left(\boldsymbol{u}_{h ; K}-\boldsymbol{u}^{\partial}\right)\left(\boldsymbol{x}_{n}\right)\right| \leq C\left(\|\boldsymbol{a}\|_{K}+h_{\gamma}\left\|\partial_{\boldsymbol{t}} \boldsymbol{u}^{\partial}-\left(\overline{\partial_{\boldsymbol{t}} \boldsymbol{u}^{\partial}}\right)_{\gamma}\right\|_{\gamma}\right) \leq C\left(\|\boldsymbol{a}\|_{K}+\underset{\gamma}{\operatorname{Osc}}\left(\partial_{\boldsymbol{t}} \boldsymbol{u}^{\partial}\right)\right) .
$$

The remainder of the argument then proceeds as in the case of homogeneous data. 


\section{Three Dimensional CASE}

To emphasize the similarities with the previous considerations, we adopt a notation analogous to the two dimensional case. We assume $\Omega$ is decomposed into a set $\mathcal{K}$ of tetrahedra satisfying the usual assumptions. Further, let $\overline{\mathcal{E}}$ be the set of all faces of tetrahedra in $\mathcal{K}$ and let $\overline{\mathcal{N}}$ be an index set for the edges. Faces and edges are assumed to be oriented in the following way: with each face $\gamma \in \overline{\mathcal{E}}$, a normal vector $\boldsymbol{n}_{\gamma}$ is assigned (which coincides with the exterior normal if $\gamma \subset \partial \Omega$ ) and each edge has a fixed direction, e.g., pointing to the vertex with the larger index in the set of vertices $\mathcal{V}:=\left\{\boldsymbol{x}_{j}\right\}_{j}$. For $\gamma \in \mathcal{E}$, let $\theta_{\gamma}$ be the linear nonconforming basis function satisfying $\theta_{\gamma}\left(\boldsymbol{x}_{\gamma^{\prime}}\right)=\delta_{\gamma \gamma^{\prime}}$, where $\boldsymbol{x}_{\gamma^{\prime}}$ is the centroid of $\gamma^{\prime}$. On the face $\gamma$ we construct an orthonormal basis $\left\{\boldsymbol{t}_{\gamma, 1}, \boldsymbol{t}_{\gamma, 2}\right\}$ satisfying $\left(\boldsymbol{t}_{\gamma, 1} \times \boldsymbol{t}_{\gamma, 2}\right) \cdot \boldsymbol{n}_{\gamma}=1$. By analogy with the two dimensional case, we define basis functions $\boldsymbol{\tau}_{\gamma, l}:=\theta_{\gamma} \boldsymbol{t}_{\gamma, l}$ and $\boldsymbol{\nu}_{\gamma}:=\theta_{\gamma} \boldsymbol{n}_{\gamma}$. The spaces $\boldsymbol{V}_{h}$ and $M_{h}$ are defined as in (2.2), and the degrees of freedom for $\boldsymbol{V}_{h}$ are identified with the functionals $f_{\gamma} \boldsymbol{v}_{h}$.

The construction of the subspace $\boldsymbol{X}_{h}$ involves choosing an appropriate subset $\mathcal{N}_{\boldsymbol{X}}$ of $\mathcal{N}$ and defining $\boldsymbol{\rho}_{n}$ as in (2.4) for $n \in \mathcal{N}_{\boldsymbol{X}}$ [18. Signs are chosen such that the face normals $\boldsymbol{\rho}_{n}\left(\boldsymbol{x}_{\gamma}\right)$ trace a path around the edge $n$ in the anti-clockwise sense with respect to the chosen orientation of this edge. The definition of $\boldsymbol{\Pi}_{h}$ remains as described in Section 2.3, and in particular, functions in $\boldsymbol{X}$ will be automatically mapped into $\boldsymbol{X}_{h}$.

We will now sketch the differences to the two dimensional case needed to extend the a posteriori error bounds in Theorems 1 and 2 under the assumption of homogeneous boundary data.

Modifications to the proof of Theorem 1. Definition 3.10(i) has to be replaced by

$$
\eta_{\mathrm{C} ; K}^{2}:=\frac{|K|}{36}\left|\overline{\boldsymbol{f}}_{K}\right|^{2} \sum_{\gamma \in \overline{\mathcal{E}}: \gamma \subset \partial K}\left|\boldsymbol{x}_{\gamma}-\boldsymbol{x}_{K}\right|^{2}
$$

for the conforming error, since we will obtain $\boldsymbol{\sigma}_{K}:=-1 / 3 \overline{\boldsymbol{f}}_{K} \otimes\left(\boldsymbol{x}-\boldsymbol{x}_{K}\right)$ for $K \in$ $\mathcal{K}$ in the proof of Theorem 1)(i), with the same technique as before (note that $\left.\left(\boldsymbol{x}-\boldsymbol{x}_{K}\right) \cdot \boldsymbol{n}_{K}=3 / 4|K| /|\gamma| \forall \boldsymbol{x} \in \gamma \subset \partial K\right)$. Part (ii) works exactly the same way.

A choice of $\boldsymbol{u}^{*}$. Before proving the lower bound, we have to be more specific about the choice of $\boldsymbol{u}^{*}$. One possibility is to choose $\boldsymbol{V}_{h}^{*}$ to be $\mathbb{P}_{\mathrm{C}}^{1}(\mathcal{K})^{3}$ enriched by face bubbles in normal directions [21, p. 122]. For this let $\theta_{\gamma}^{*}$ be the cubic face bubble for $\gamma \in \overline{\mathcal{E}}$ with $f_{\gamma} \theta_{\gamma}^{*}=1$. For each vertex $\boldsymbol{x}_{j}$ let $\phi_{j}$ its piecewise linear basis function and let $\boldsymbol{S}\left(\boldsymbol{v}_{h}\right)_{j}$ be the weighted mean for $\boldsymbol{v}_{h} \in \boldsymbol{V}_{h}$ as in (3.11). We then define $\boldsymbol{P}_{h}: \boldsymbol{V}_{h} \rightarrow \boldsymbol{V}_{h}^{*}$ by $\boldsymbol{P}_{h} \boldsymbol{v}_{h}:=\sum_{j \in \mathcal{V}} \boldsymbol{S}\left(\boldsymbol{v}_{h}\right)_{j} \phi_{j}+\sum_{\gamma \in \overline{\mathcal{E}}} T\left(\boldsymbol{v}_{h}\right)_{\gamma} \theta_{\gamma}^{*} \boldsymbol{n}_{\gamma}$, where $T(.)_{\gamma}$ is chosen such that $\int_{\gamma} \boldsymbol{P}_{h} \boldsymbol{v}_{h} \cdot \boldsymbol{n}_{\gamma}=\int_{\gamma} \boldsymbol{v}_{h} \cdot \boldsymbol{n}_{\gamma}$. Thus we obtain that for $\boldsymbol{v}_{h} \in \boldsymbol{X}_{h}$, (3.13) holds for piecewise constant $q_{h}$ and, therefore, $M_{h}^{*}=M_{h}=\mathbb{P}^{0}(\mathcal{K})$. Our choice here is $\boldsymbol{u}^{*}:=\boldsymbol{P}_{h} \boldsymbol{u}_{h}$.

Modifications to the proof of Theorem 2(i). Taking $\boldsymbol{v}:=\boldsymbol{\nabla} \times \boldsymbol{r}_{l}$, where $\boldsymbol{r}_{l}:=s \boldsymbol{t}_{\gamma, l}$ for some $s \in C_{0}^{2}\left(\Omega_{\gamma}\right)$ and $l \in\{1,2\}$, we achieve $\boldsymbol{\nabla} \cdot \boldsymbol{v}=0$. Now we obtain, for some $c_{\gamma}>0$

$$
\int_{\Omega} \boldsymbol{\sigma}: \nabla \boldsymbol{v}=\sum_{K \in\left\{K^{\prime}, K^{\prime \prime}\right\}}\left\{-c_{\gamma} \overline{\boldsymbol{f}}_{K} \cdot \int_{\gamma} \boldsymbol{\nabla} s \times \boldsymbol{t}_{\gamma, l}+\overline{\boldsymbol{f}}_{K} \cdot\left(\boldsymbol{n}_{\gamma} \times \boldsymbol{t}_{\gamma, l}\right) \int_{\gamma} s\right\} .
$$


Using $\left\{l, l^{\prime}\right\}=\{1,2\}$ and cyclic permutation, we see that

$$
\int_{\gamma} \boldsymbol{\nabla} s \times \boldsymbol{t}_{\gamma, l}=\int_{\gamma}\left(\boldsymbol{\nabla} s \times \boldsymbol{t}_{\gamma, l}\right) \cdot \boldsymbol{t}_{\gamma, l^{\prime}} \boldsymbol{t}_{\gamma, l^{\prime}} \sim \int_{\gamma} \partial_{\boldsymbol{n}} s \boldsymbol{t}_{\gamma, l^{\prime}}
$$

and $\overline{\boldsymbol{f}}_{K} \cdot\left(\boldsymbol{n}_{\gamma} \times \boldsymbol{t}_{\gamma, l}\right) \sim \overline{\boldsymbol{f}}_{K} \cdot \boldsymbol{t}_{\gamma, l^{\prime}}$. Hence, we end up in the same situation as in (3.17) and, with the same choice of $s$ as before, one obtains an estimate for the tangential part of $\overline{\boldsymbol{f}}_{K}$ on $\gamma$ and, using a second face of $K$, an estimate for the whole vector.

Modifications to the proof of Theorem $2\left(\right.$ ii.1). For $l \in\{1,2\}$ let $\boldsymbol{v}_{i}:=z_{i} \boldsymbol{t}_{\gamma, l^{\prime}} \theta_{\gamma}^{*}$ for some $\boldsymbol{z} \in \mathbb{R}^{3}, i \in\{1,2,3\}, l^{\prime}$ such that $\left\{l, l^{\prime}\right\}=\{1,2\}$. Define $\boldsymbol{w}(\boldsymbol{x})$ to be the matrix with $i$ th row $\boldsymbol{\nabla} \times \boldsymbol{v}_{i}(\boldsymbol{x})$. Then $\boldsymbol{\nabla} \cdot \boldsymbol{w}=0$ so that $\boldsymbol{w} \in \boldsymbol{Y}$, and one obtains

$$
\begin{aligned}
\int_{\Omega} \boldsymbol{a}: \boldsymbol{w} & =-\int_{\Omega} \sum_{i=1}^{3} \boldsymbol{\nabla}_{h} u_{h, i} \cdot \boldsymbol{\nabla} \times \boldsymbol{v}_{i}=-\sum_{i=1}^{3}\left[\boldsymbol{\nabla} u_{h, i}\right]_{\gamma} \cdot\left(\boldsymbol{n}_{\gamma} \times \boldsymbol{t}_{\gamma, l^{\prime}}\right) z_{i} h_{\gamma} \\
& \sim-\left[\partial_{\boldsymbol{t}_{\gamma, l}} \boldsymbol{u}_{h}\right]_{\gamma} \cdot \boldsymbol{z} h_{\gamma} .
\end{aligned}
$$

Choosing $\boldsymbol{z}=\left[\partial_{\boldsymbol{t}_{\gamma, l}} \boldsymbol{u}_{h}\right]_{\gamma}$ yields the required bound.

Modifications in the proof of Theorem 2(ii.2). The essential steps are to show that $\boldsymbol{P}_{h} \boldsymbol{u}_{h L_{K}}$ depends on coefficients of $\boldsymbol{u}_{h}$ on $\Omega_{K}$ and that vanishing jumps $J_{\boldsymbol{t} ; \gamma}$ for $\gamma \in \mathcal{A}_{K}$ imply $\boldsymbol{u}^{*}=\boldsymbol{u}_{h}$ on $K$. The first property is immediate. For the second we show that, as before, $\boldsymbol{u}_{h}$ is continuous on $\Omega_{K}$ and thus equals $\boldsymbol{u}^{*}$ at all vertices. Since $\boldsymbol{\nabla} \cdot \boldsymbol{u}_{h}=0$ we see that $T\left(\boldsymbol{u}_{h}\right)_{\gamma}=0$ for all $\gamma \subset \partial K$ and, therefore, $\boldsymbol{u}^{*}=\boldsymbol{u}_{h}$ on $K$.

\section{Numerical EXAMPleS}

We show numerical results for two examples. The first one has a polynomial velocity field of third order on $\Omega:=(0,1)^{2}$ with nonhomogeneous data $\boldsymbol{f}$ and $\boldsymbol{u}^{\partial}$. Velocity, stream function, and pressure, respectively, are given by

$$
\begin{aligned}
\boldsymbol{u}_{e}\left(x_{1}, x_{2}\right) & =\left[x_{1}\left(1-x_{1}\right)\left(1-2 x_{2}\right),-x_{2}\left(1-x_{2}\right)\left(1-2 x_{1}\right)\right], \\
\Psi_{e}\left(x_{1}, x_{2}\right) & =x_{1} x_{2}\left(1-x_{1}\right)\left(1-x_{2}\right), \\
p_{e}\left(x_{1}, x_{2}\right) & =2\left(x_{2}-x_{1}\right) .
\end{aligned}
$$

The second example (see, e.g., [26]) has a singular solution $\left(\boldsymbol{u}_{e} \notin H^{2}(\Omega)^{2}\right)$ on the L-shaped domain $\Omega:=(-1,1)^{2} \backslash(0,1) \times(-1,0)$ with $\boldsymbol{f}=\mathbf{0}$, explicitly given by

$$
\begin{aligned}
\boldsymbol{u}_{e}(r, \phi)=r^{\alpha}\left[(\alpha+1) \sin (\phi) \psi(\phi)+\cos (\phi) \psi^{\prime}(\phi),\right. & \\
& \left.\quad-(\alpha+1) \cos (\phi) \psi(\phi)+\sin (\phi) \psi^{\prime}(\phi)\right], \\
\Psi_{e}(r, \phi)= & r^{\alpha+1} \psi(\phi), \\
p_{e}(r, \phi)= & \frac{1}{\alpha-1} r^{\alpha-1}\left((\alpha+1)^{2} \psi^{\prime}(\phi)+\psi^{\prime \prime \prime}(\phi)\right)
\end{aligned}
$$

in polar coordinates $[r, \phi] \in(0, \infty) \times(0,3 \pi / 2)$, where

$$
\begin{aligned}
\psi(\phi)= & \frac{1}{\alpha+1} \sin ((\alpha+1) \phi) \cos (\alpha \omega)-\cos ((\alpha+1) \phi), \\
& +\frac{1}{\alpha-1} \sin ((\alpha-1) \phi) \cos (\alpha \omega)+\cos ((\alpha-1) \phi), \\
\alpha= & \frac{856399}{1572864} \approx 0.54, \quad \omega=3 \pi / 2 .
\end{aligned}
$$


TABLe 1. Results for Example 1 on the uniform grid with $N=$ 3969 (left) and for Example 2 on the uniform grid with $N=5985$ (right) for the different choices of $\boldsymbol{Q}_{h}$.

\begin{tabular}{|r|r|r|r|}
\hline $\boldsymbol{Q}_{h}$ & $\bar{\eta}$ & $\bar{\eta}^{(u)}$ & $\bar{\eta}^{(d)}$ \\
\hline $\boldsymbol{Q}_{h}^{0}$ & $1.73_{-1}$ & $5.11_{-2}$ & $3.49_{-2}$ \\
$\boldsymbol{Q}_{h}^{\text {DDF }}$ & $2.02_{-1}$ & $9.66_{-2}$ & $2.84_{-2}$ \\
$\boldsymbol{Q}_{h}^{\mathrm{MIN}}$ & $1.55_{-1}$ & $6.31_{-2}$ & $2.31_{-2}$ \\
$\boldsymbol{Q}_{h}^{\text {OPT }}$ & $1.55_{-1}$ & $5.77_{-2}$ & $2.51_{-2}$ \\
\hline
\end{tabular}

\begin{tabular}{|r|r|r|r|}
\hline $\boldsymbol{Q}_{h}$ & $\bar{\eta}$ & $\bar{\eta}^{(u)}$ & $\bar{\eta}^{(d)}$ \\
\hline $\boldsymbol{Q}_{h}^{0}$ & $3.37_{-1}$ & $1.05_{-1}$ & $6.98_{-2}$ \\
$\boldsymbol{Q}_{h}^{\mathrm{DDF}}$ & $3.69_{-1}$ & $1.81_{-1}$ & $5.63_{-2}$ \\
$\boldsymbol{Q}_{h}^{\mathrm{MIN}}$ & $2.77_{-1}$ & $1.24_{-1}$ & $4.60_{-2}$ \\
$\boldsymbol{Q}_{h}^{\text {OPT }}$ & $2.77_{-1}$ & $1.18_{-1}$ & $4.79_{-2}$ \\
\hline
\end{tabular}

In the experiments we calculate the "exact" (using a quadrature formula for $\mathbb{P}^{2}$ ) and the estimated error in the energy norm $\| \boldsymbol{\nabla}$. $\|$ (using a quadrature formula for $\mathbb{P}^{3}$ ) for the velocity on a sequence of uniformly and adaptively refined grids, respectively. For each marked triangle two newest node bisection steps [27] were performed. As a local error indicator for the adaptive algorithm we used (cf. Section 3.3.

$$
\eta_{K}^{2}:=\eta_{\mathrm{C} ; K}^{2}+\left\|\boldsymbol{\nabla}\left(\boldsymbol{u}^{*}-\boldsymbol{u}_{h}\right)\right\|_{K}^{2}+\frac{1}{c_{0}^{2}}\left\|\boldsymbol{\nabla} \cdot \boldsymbol{u}^{*}\right\|_{K}^{2},
$$

and triangles are marked using the maximum strategy (mark $K$ if $\eta_{K} \geq \eta_{\max } / 2$ ). The global error estimate is, according to (3.10), given by

$$
\eta:=\eta_{\mathrm{C}}+\eta_{\mathrm{NC}} .
$$

Values for the constant $c_{0}=0.4$ for $\Omega=(0,1)^{2}$ and $c_{0}=0.3$ for the L-shaped domain have been obtained from [25, pp. 253-254]. All errors will be presented as relative errors. For convenience, the following abbreviations will be used: $E:=$ $\left\|\boldsymbol{\nabla}\left(\boldsymbol{u}_{e}-\boldsymbol{u}_{h}\right)\right\| /\left\|\boldsymbol{\nabla} \boldsymbol{u}_{e}\right\|, \bar{\eta}:=\eta /\left\|\boldsymbol{\nabla} \boldsymbol{u}_{e}\right\|, \bar{\eta}^{(u)}:=\left\|\boldsymbol{\nabla}\left(\boldsymbol{u}^{*}-\boldsymbol{u}_{h}\right)\right\| /\left\|\boldsymbol{\nabla} \boldsymbol{u}_{e}\right\|, \bar{\eta}^{(d)}:=$ $\left\|\boldsymbol{\nabla} \cdot \boldsymbol{u}^{*}\right\| /\left\|\boldsymbol{\nabla} \boldsymbol{u}_{e}\right\|$.

Table 1 compares the bounds obtained using the four different types of postprocessing scheme used to define $\boldsymbol{u}^{*}$ in the case of a uniform grid. Observe that the estimators involving $\boldsymbol{Q}_{h}^{\mathrm{OPT}}$ and $\boldsymbol{Q}_{h}^{\mathrm{MIN}}$ perform comparably and represent a significant improvement over the estimators based on $\boldsymbol{Q}_{h}^{0}$ and $\boldsymbol{Q}_{h}^{\mathrm{DDF}}$ in this example.

Figure 1 shows the convergence history for both examples on a sequence of uniformly and adaptively refined grids; Tables 2 and 3 , the corresponding data. In the first example, which is $H^{2}$-regular, the slope of the lines is about $-1 / 2$, confirming the expected relations $E \sim N^{-1 / 2}$ and $\bar{\eta} \sim N^{-1 / 2}$, respectively. However, the exact error is overestimated by a factor of about 3.5. In the second example the slope of the data in the uniform case is about $-1 / 4$, which is consistent with the expected rate of $-\alpha / 2$. The adaptive algorithm improves upon this by that the error decreases with $N^{-1 / 2}$ as in the $H^{2}$-regular case. This confirms that the adaptive algorithm is quasi-optimal, although, once again, there is overestimation by a factor of about 3 in the uniform case and approximately 3.9 in the adaptive case.

The cost of using $\boldsymbol{Q}_{h}^{\mathrm{OPT}}$ is about $10 \%$ more than that for $\boldsymbol{Q}_{h}^{0}$. Pressure errors are of the size of the velocity errors, and the overestimation of $\eta$ with respect to $\left\|\boldsymbol{\nabla}\left(\boldsymbol{u}_{e}-\boldsymbol{u}_{h}\right)\right\|+c_{0}\left\|p_{e}-p_{h}\right\|$ is about a factor of 3 . 

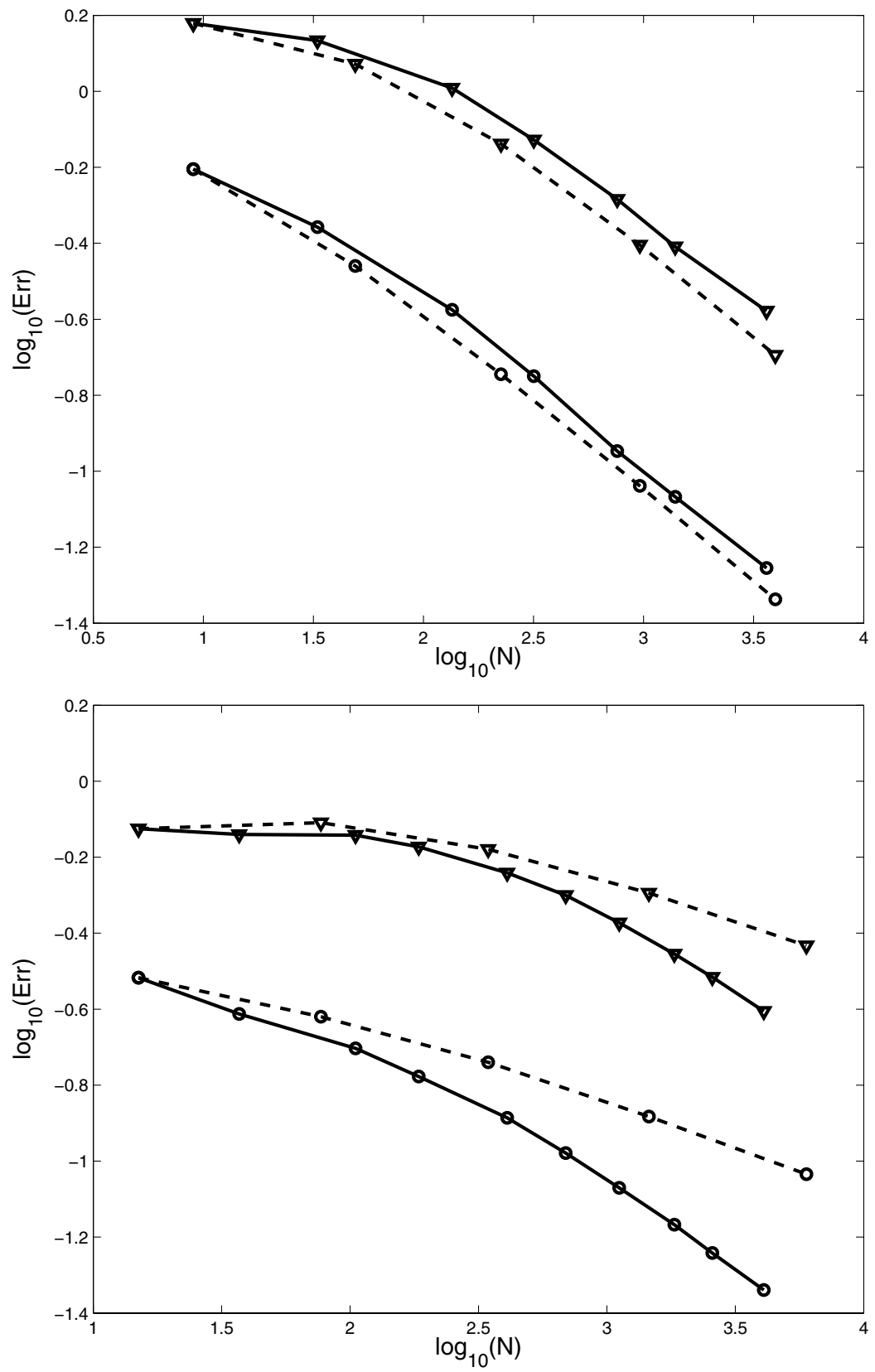

Figure 1. Exact (o) and estimated error $(\nabla)$ on uniform grids (dashed) and adaptive grids (solid) for the first example (top) and the second example (bottom). 
TABLe 2. Data for the uniform (left) and adaptive (right) results in Figure 1 (Example 1) using $\boldsymbol{u}^{*}=\boldsymbol{Q}_{h}^{\mathrm{OPT}} \boldsymbol{u}_{\boldsymbol{h}}$.

\begin{tabular}{|r|l|l|c|}
\hline$N$ & $E$ & $\bar{\eta}$ & $\bar{\eta} / E$ \\
\hline 9 & $6.24_{-1}$ & 1.44 & 2.31 \\
49 & $3.47_{-1}$ & $1.01_{1}$ & 2.91 \\
225 & $1.80_{-1}$ & $5.81_{-1}$ & 3.23 \\
961 & $9.15_{-2}$ & $3.05_{-1}$ & 3.33 \\
3969 & $4.60_{-2}$ & $1.55_{-1}$ & 3.37 \\
\hline
\end{tabular}

\begin{tabular}{|r|r|l|c|}
\hline$N$ & $E$ & $\bar{\eta}$ & $\bar{\eta} / E$ \\
\hline 9 & $6.24_{-1}$ & 1.44 & 2.31 \\
47 & $3.74_{-1}$ & 1.08 & 2.89 \\
173 & $2.16_{-1}$ & $7.04_{-1}$ & 3.26 \\
329 & $1.69_{-1}$ & $5.71_{-1}$ & 3.38 \\
991 & $9.69_{-2}$ & $3.43_{-1}$ & 3.54 \\
1287 & $8.60_{-2}$ & $2.99_{-1}$ & 3.48 \\
4537 & $4.63_{-2}$ & $1.64_{-1}$ & 3.54 \\
\hline
\end{tabular}

TABLE 3. Data for the uniform (left) and adaptive (right) results in Figure 1 (Example 2) using $\boldsymbol{u}^{*}=\boldsymbol{Q}_{h}^{\mathrm{OPT}} \boldsymbol{u}_{h}$.

\begin{tabular}{|r|r|r|r|}
\hline$N$ & $E$ & $\bar{\eta}$ & $\bar{\eta} / E$ \\
\hline 15 & $3.04_{-1}$ & $6.04_{-1}$ & 1.99 \\
77 & $2.40_{-1}$ & $6.13_{-1}$ & 2.55 \\
345 & $1.82_{-1}$ & $5.12_{-1}$ & 2.81 \\
1457 & $1.31_{-1}$ & $3.86_{-1}$ & 2.95 \\
5985 & $9.24_{-2}$ & $2.77_{-1}$ & 3.00 \\
\hline
\end{tabular}

\begin{tabular}{|r|r|r|c|}
\hline$N$ & $E$ & $\bar{\eta}$ & $\bar{\eta} / E$ \\
\hline 15 & $3.04_{-1}$ & $6.04_{-1}$ & 1.99 \\
37 & $2.44_{-1}$ & $5.78_{-1}$ & 2.37 \\
105 & $1.98_{-1}$ & $5.62_{-1}$ & 2.84 \\
177 & $1.68_{-1}$ & $5.16_{-1}$ & 3.07 \\
319 & $1.38_{-1}$ & $4.60_{-1}$ & 3.33 \\
585 & $1.11_{-1}$ & $3.89_{-1}$ & 3.50 \\
911 & $9.19_{-2}$ & $3.33_{-1}$ & 3.62 \\
1455 & $7.45_{-2}$ & $2.79_{-1}$ & 3.75 \\
2343 & $5.98_{-2}$ & $2.27_{-1}$ & 3.80 \\
3369 & $5.02_{-2}$ & $1.95_{-1}$ & 3.88 \\
\hline
\end{tabular}

\section{REFERENCES}

1. A. Agouzal, A posteriori error estimator for non-conforming finite element methods, Appl. Math. Lett. 7 (1994), 1017-1033. MR1350612

2. M. Ainsworth, Robust a posteriori error estimates for non-conforming finite element approximation, SIAM J. Numer. Anal., to appear.

3. T. Apel, S. Nicaise, and J. Schöberl, A non-conforming finite element method with anisotropic mesh grading for the Stokes problem in domains with edges, IMA J. Numer. Anal. 21 (2001), 843-856. MR1867421 (2003a:65100)

4. W. Z. Bao and J. W. Barrett, A priori and a posteriori error bounds for a nonconforming linear finite element approximation of a non-Newtonian flow, RAIRO Modél. Math. Anal. Numér. 32 (1998), 843-858. MR1654432 (99i:76086)

5. F. Brezzi and M. Fortin, Mixed and hybrid finite element methods, Springer, New York, 1991. MR.1115205 (92d:65187)

6. C. Carstensen and S. A. Funken, Fully realiable localised error control in the FEM, SIAM J. Sci. Comput. 21 (2000), 1465-1484. MR.1742328 (2000k:65205)

7. _ A posteriori error control in low-order finite element discretisations of incompressible stationary flow problems, Math. Comput. 70 (2001), 1353-1381. MR.1836908 (2002f:65157)

8. E. V. Chizhonkov and M. A. Olshanskii, On the domain geometry dependence of the LBB condition, RAIRO Modél. Math. Anal. Numér. 34 (2000), 935-951. MR1837762(2002c:65203)

9. M. Crouzeix and P. A. Raviart, Conforming and nonconforming finite element methods for solving the stationary Stokes equations, RAIRO Anal. Numér. 7 (1973), 33-76. MR0343661 (49:8401) 
10. E. Dari, R. G. Durán, and C. Padra, Error estimators for nonconforming finite element approximations of the Stokes problem, Math. Comput. 64 (1995), 1017-1033. MR.1284666 (95j:65136)

11. , Maximum norm error estimators for three-dimensional elliptic problems, SIAM J. Numer. Anal. 37 (2000), 683-700. MR1740762 (2001b:65120)

12. E. Dari, R. G. Durán, C. Padra, and V. Vampa, A posteriori error estimators for nonconforming finite element methods, RAIRO Modél. Math. Anal. Numér. 30 (1996), 385-400. MR.1399496 (97f:65066)

13. Q. P. Deng, X. Xu, and S. Shen, Maximum norm error estimates of Crouzeix-Raviart nonconforming finite element approximation of Navier-Stokes problem, J. Comput. Math. 18 (2000), 141-156. MR 1750943 (2001d:65129)

14. R. G. Durán and C. Padra, An error estimator for nonconforming approximations of a nonlinear problem, Finite element methods, fifty years of the Courant element (M. Krizek, P. Neittaanmaki, and R. Stenberg, eds.), Dekker, New York, 1994, pp. 201-205. MR.1299990

15. M. Fortin, Utilisation de la méthode des éléments finis en mécanique des fluides. I, Calcolo 12 (1975), 405-441. MR0421339(54:9344a)

16. G. P. Galdi, An Introduction to the Mathematical Theory of the Navier-Stokes Equations I, Springer, New York, 1994. MR1284205(95i:35216a)

17. D. F. Griffiths, Finite elements for incompressible flows, Math. Methods Appl. Sci. 1 (1979), 16-31. MR0548403 (80j:76027)

18. F. Hecht, Construction d'une base de fonctions $P 1$ non conforme à divergence nulle dans R3, RAIRO Anal. Numér. 15 (1981), 119-150. MR0618819 (83j:65023)

19. C. Padra, A posteriori error estimators for nonconforming approximation of some quasiNewtonian flows, SIAM J. Numer. Anal. 34 (1997), 1600-1615. MR1461798 (98h:65050)

20. M. Paraschivoiu and A. T. Patera, A posteriori bounds for linear functional outputs of Crouzeix-Raviart finite element discretizations of the incompressible Stokes problem, Internat. J. Numer. Methods Fluids 32 (2000), 823-849. MR.1752470(2001e:76088)

21. R. Pierre, Local mass conservation and $C^{0}$-discretisations of the Stokes problem, Houston J. 20 (1994), 115-127. MR.1272565 (95c:65167)

22. V. Ruas, Finite element solution of $3 D$ viscous flow problems using nonstandard degrees of freedom, Japan J. Appl. Math. 2 (1985), 415-431. MR0839337 (87m:65191)

23. __ Circumventing discrete Korn's inequalities in convergence analyses of nonconforming finite element approximations of vector fields, Z. Angew. Math. Mech. 76 (1996), 483-484. MR:1409361 (97h:73079)

24. F. Schieweck and L. Tobiska, An optimal order error estimate for an upwind discretization of the Navier-Stokes equations, Numer. Meth. PDE 12 (1996), 407-421. MR.1396464 (97c:65193)

25. G. Stoyan, Towards discrete Velte decompositions and narrow bounds for inf-sup constants, Comput. Math. Appl. 38 (1999), 243-261. MR1713178 (2000k:65154)

26. R. Verfürth, A posteriori error estimators for the Stokes equations, Numer. Math. 55 (1989), 309-325. MR0993474 (90d:65187)

27. — A Review of A Posteriori Error Estimation and Adaptive Mesh-Refinement Techniques, Wiley-Teubner, Chichester, 1996.

Institut Für Angewandte Mathematik II, Univ. Karlsruhe, 76128 Karlsruhe, Germany E-mail address: doerfler@mathematik.uni-karlsruhe.de

Department of Mathematics, Strathclyde University, 26 Richmond St., Glasgow G1 $1 \mathrm{XH}$, SCOTLAND

E-mail address: M.Ainsworth@strath.ac.uk 